\title{
Effective adjustment of the optoelectronic properties of organic conjugated materials by synthesizing $p$-n diblock molecules
}

\author{
JIANG HongJi ${ }^{1}$ \\ Key Laboratory of Organic Electronics \& Information Displays, Institute of Advanced Materials, Nanjing University of Posts and \\ Telecommunications, Nanjing 210046, China
}

Received March 25, 2010; accepted June 22, 2010

\begin{abstract}
Because organic conjugated materials offer several advantages relative to their inorganic counterparts, the development of organic conjugated materials has been one of the most active research areas in optoelectronic materials. For almost two decades, the search for organic conjugated materials has represented a major driving force for research concerned with controlling the band gap of extended $\pi$-conjugated molecules. In particular, among the parameters affecting the performance of organic light-emitting diodes (OLEDs), the energy levels of organic conjugated materials play an important role because they can affect the driving voltage, wavelength, efficiency, and lifetime of the final device. Balanced injection and transport of electrons and holes are therefore crucial for achieving OLEDs with high quantum efficiency. In this regard, research into adjusting the energy levels of organic conjugated materials is very meaningful for the development of OLEDs. To adjust the energy levels of the organic conjugated materials, Huang et al. have presented a new molecular design and synthesis route that yields $p-n$ diblock conjugated copolymers and oligomers. The present review summarizes and analyzes the progress on adjusting the optoelectronic properties of organic conjugated materials that is due to synthesizing $p$ - $n$ diblock molecules. We discusses primarily work done by Huang et al., but also discusses work done elsewhere over the past few years. We also point out issues that require attention, and highlight hot spots that require further investigation.
\end{abstract}

adjust, diblock, energy levels, organic light-emitting diodes, synthesize

Citation: Jiang H J. Effective adjustment of the optoelectronic properties of organic conjugated materials by synthesizing $p-n$ diblock molecules. Chinese Sci Bull, 2011, 56: 119-136, doi: 10.1007/s11434-010-4246-5

Information display technologies are widely applied in the entertainment, industry and military sectors and are becoming one of the key foundations of the optoelectronic industry. Liquid crystal displays (LCDs), first used in the 1980s, are now the main tideway among the flat-panel display technologies. However, the dominant position of LCDs in market is being gradually challenged by flexible information display technologies such as organic light-emitting diodes (OLEDs) because of their easy processability, low turn-on voltage, wide viewing angle, and facile color tunability over the full visible range. With major efforts from both industry and academia, significant advancements have been made over the past few decades in this new flexible

*Corresponding author (email: iamhjjiang@ njupt.edu.cn) information display technology [1-5]. The simplest OLED sandwiches the emissive layer between two electrodes. At the cathode, electrons are injected into the lowest unoccupied molecular orbital (LUMO) and, at the anode, holes are injected into the highest occupied molecular orbital (HOMO). Under the applied bias, the two opposite charges move through the layer and, if they meet in the same material, form an exciton that can photodecay by emitting light at a wavelength determined by the band gap of the material. Therefore, for an efficient OLED, it is necessary to have balanced charge injection and transport, capture all the injected charge to form excitons, and have all the excitons to decay radiatively [6]. In this regard, the energy levels of the optoelectronic materials play an important role in determining the performance of OLEDs, so controlling the HOMO 
and LUMO energy levels of $\pi$-conjugated molecules (and thereby the band gap of the corresponding bulk material) has been at the center of the synthetic chemistry of functional $\pi$-conjugated systems for more than 20 years $[7,8]$. Huang et al. present a new molecular design and synthesis in which they incorporate typical $p$-doped and $n$-doped segments into a single conjugated polymer backbone. The present review summarizes and analyzes progress made in adjusting the optoelectronic properties of organic conjugated materials as a result of synthesizing $p$ - $n$ diblock molecules. We concentrate primarily on the work done by Huang et al. over the past few years. Finally, strategies that have proven successful are highlighted to improve our understanding of $p-n$ diblock conjugated materials.

\section{Synthesizing linear $p-n$ diblock polymers to adjust the energy levels of organic conjugated materials}

Because balanced injection and transport of electrons and holes are essential for achieving highly efficient and stable OLEDs, it is desirable to develop new electroluminescent (EL) polymers with intrinsically balanced electron and hole injection and transport. To achieve this goal, the key is to develop a synthesis route that allows effective adjustment of the HOMO and LUMO energy levels of conjugated polymers. Huang et al. have presented a new molecular design and synthesis route to form $p$ - $n$ diblock conjugated copolymers, in which typical $p$-doped and $n$-doped segments are alternately incorporated into backbones of the conjugated polymers (Figure 1). This strategy allows the adjustment of the HOMO and LUMO energy levels and thus of the emissive color of the resulting polymers 1-7. The results of electrochemical studies [9] indicate that this synthetic route is a promising method to adjust the HOMO and LUMO energy levels of EL polymers in order to balance the injection of electrons and holes from opposing contacts. On the basis of this research, Huang et al. synthesized conjugated polymers $\mathbf{8}$ and $\mathbf{9}$, which are composed of 3-alkylthiophenes and 1,4-di(1,3,4-oxadiazolyl)phenylene (Figure 2), and discussed the effects of the length of the substituted chains of the thiophene (THP) ring on the optoelectronic properties of the polymers. The solubility of the precursor polymers and the corresponding target polymers are improved by increasing the side-chain length on the THP rings, which results in a higher degree of conversion from the precursor to the final polymer and thus results in longer effective conjugation length in the polymers with longer side chains. The relative photoluminescent (PL) yield in solution increases with increasing side-chain length for THP rings in the polymers and reaches $79 \%$ for polymers bearing octyl side chains [10]. Guided by this synthesis strategy, Huang et al. synthesized copolymers 10, 11, and 12 based on 1,4-bis(1,3,4oxadiazol-2-yl)-2,5-dialkoxybenzene and oligothiophene
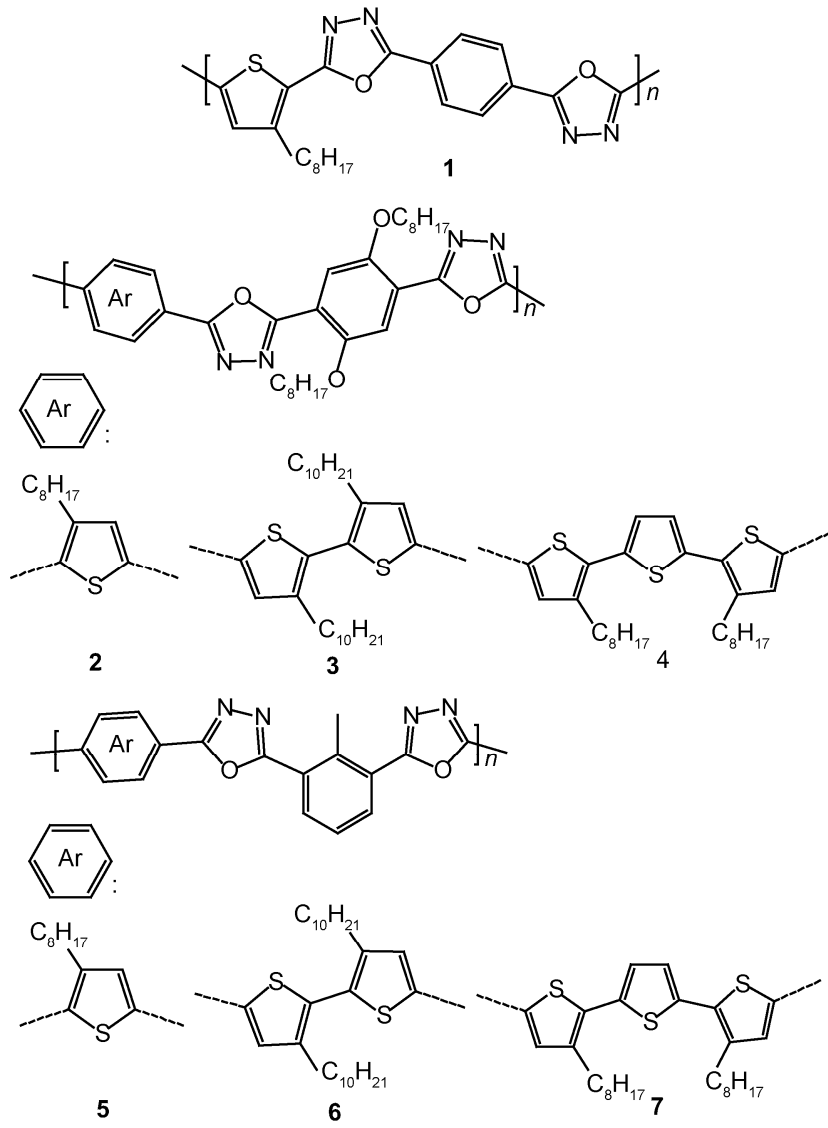

Figure 1

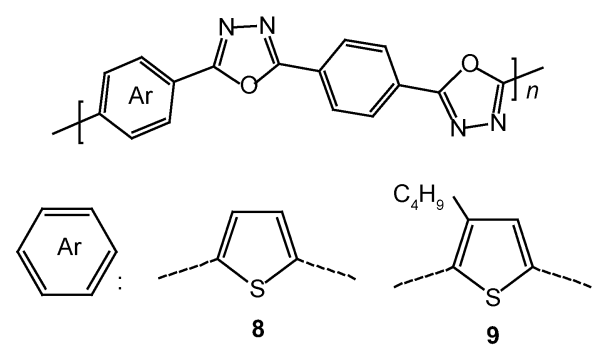

Figure 2

(Figures 3 and 4). The emissive color of the copolymers is tunable from blue $(489 \mathrm{~nm})$, green $(530 \mathrm{~nm})$ to orange (579 $\mathrm{nm}$ ) by increasing the number of THP rings from one to three in the oligothiophene blocks [11-13].

Apart from polymers based on THP and oxadiazole (OXD), Huang et al. extended the $p$ - $n$ diblock molecular

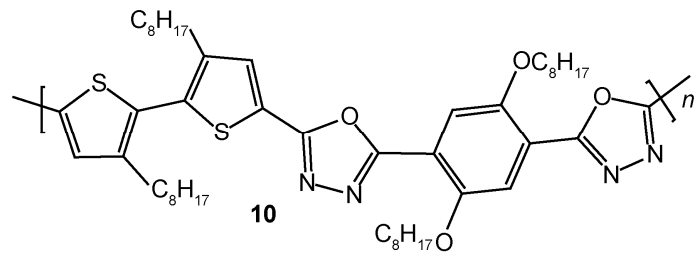

Figure 3 


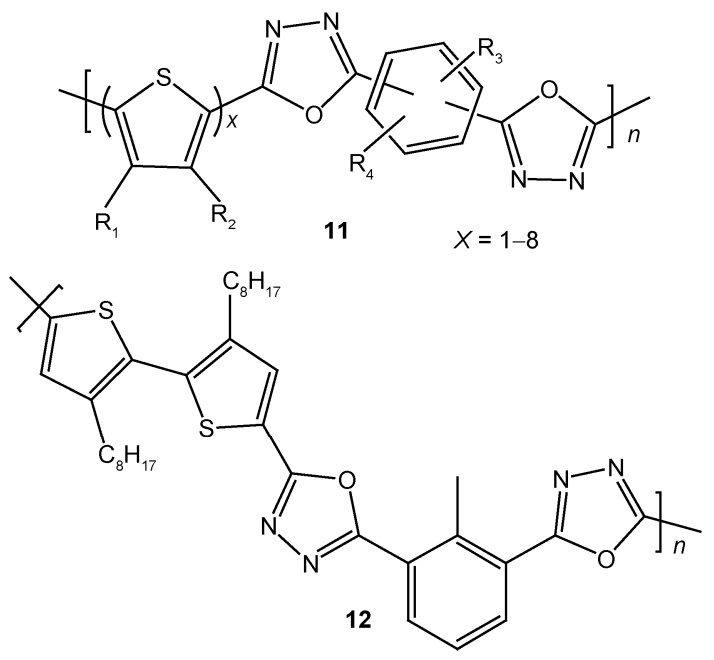

Figure 4

strategy to other systems. They synthesized a novel $p$ - $n$ diblock copolymer 13, which is composed of an electron-rich moiety carbazole (CBZ) and an electron-deficient aromatic OXD to balance the injection and transport of holes and electrons (Figure 5) [14]. Electrochemical analyses by cyclic voltammetry $(\mathrm{CV})$ indicate that the polymer can be reversibly $n$-doped and irreversibly $p$-doped. The cathodic sweep reveals that reduction involves a two-elec- tron process with respect to the successive reduction of OXD rings and $\mathrm{CBZ}$ moieties in the polymer chain. From the onset of the oxidation and reduction potentials, the HOMO and LUMO energy levels of polymer $\mathbf{1 3}$ are estimated to be -5.60 and $-2.66 \mathrm{eV}$, respectively, so this polymer has a blue-green emission (475 $\mathrm{nm})$. The HOMO and LUMO energy levels of the polymer are adjusted by combining the electron-deficient OXD moiety and the electron-rich CBZ segment into the polymer backbone [15-17], which improves the imbalanced hole-electron injection or transporting ability of most conjugated polymers encountered in OLEDs [18-22].

The discovery of electroluminescence based on $\operatorname{poly}(p$ phenylenevinylene) (PPV) triggered enormous research

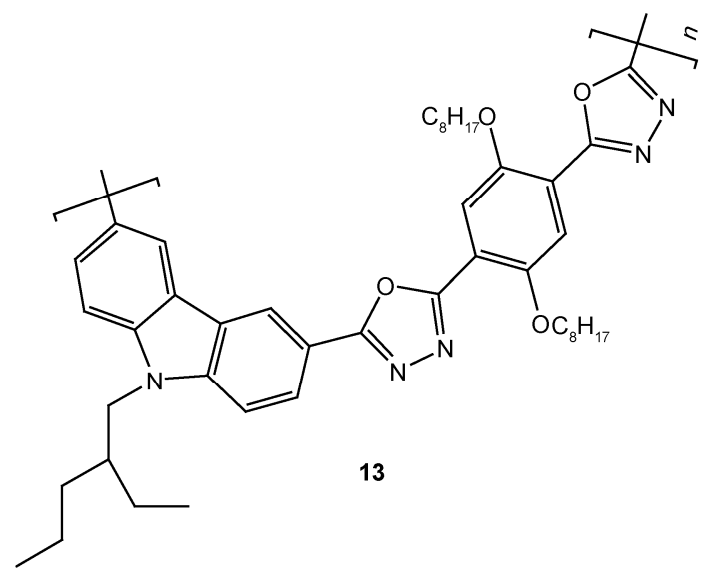

Figure 5 activities in the field of conjugated polymers for applications in OLEDs. Because most conjugated polymers such as PPV are better suited for hole injection and transport compared with electron injection and transport, the unsatisfactory quantum efficiency of OLEDs is attributed to the lower injection rate of electrons from the cathode into the polymer emissive layer. On the basis of the $p$ - $n$ diblock concept, Huang et al. synthesized a conjugated polymer consisting of 2,2'-bipyridylenevinylene (BPyV) and 2-meth-oxy-5(2-ethylhexyloxy)-1,4-phenylenevinylene (MEH-PV) moieties by incorporating the $n$-doped moiety $\mathbf{B P y V}$ and the p-doped moiety MEH-PV into the polymer chain 14 (Figure 6). The HOMO (LUMO) energy levels of 14, 15, and 16 were $-5.18(-2.81) \mathrm{eV},-5.67(-2.79) \mathrm{eV}$, and -4.99 $(-2.71)$ eV [23]. Polymer 14 exhibits tunable optoelectronic properties through protonation, and the emissive color can be progressively changed from orange to deep red by changing the degree of protonation. Huang et al. synthesized copolymers 17 containing 2,5-dicyano-1,4-phenylenevinylene and 2-methoxy-5-(2'-ethylhexyloxy)-1,4-phenylenevinylene using the Wittig reaction (Figure 7). The HOMO and LUMO energy levels of copolymer $\mathbf{1 7}$ are in the range of -5.5 to $-4.8 \mathrm{eV}$ and -3.58 to $-2.7 \mathrm{eV}$, respectively, which are easily tuned over a range of 0.7 to $0.8 \mathrm{eV}$. The polymer can be changed from a typical hole-transport (HT) material to a typical electron-transport (ET) material by varying the monomer feed ratio [24]. Quantum chemical calculations and previous research have demonstrated that introducing an electron-withdrawing group onto either the aryl ring or the vinyl group of PPV can lower both the HOMO and LUMO energy levels of the polymer, thus improving electron injection. Previous research has demonstrated the feasibility of tuning the optoelectronic properties of $p-n$ diblock conjugated structures containing the OXD moiety. Along these lines, Huang et al. synthesized the two PPV-based polymers 18 and 19, which are functionalized

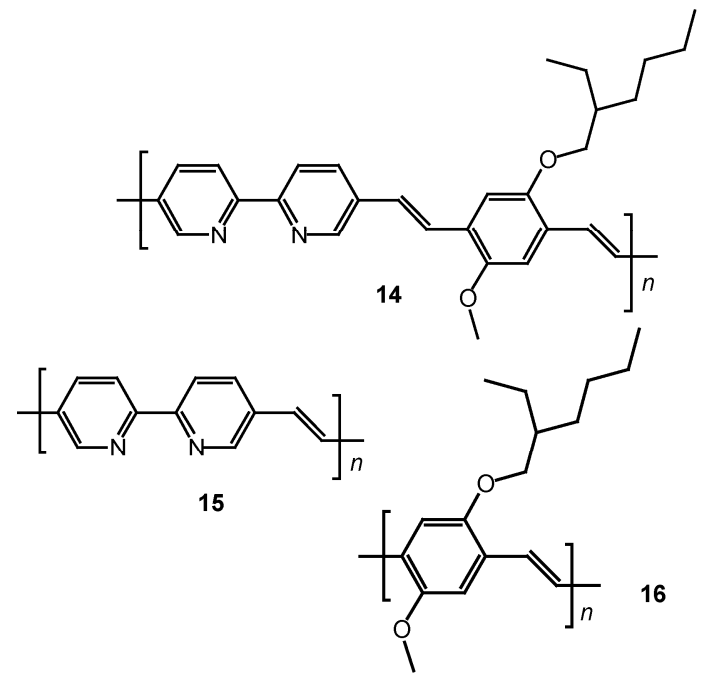

Figure 6 


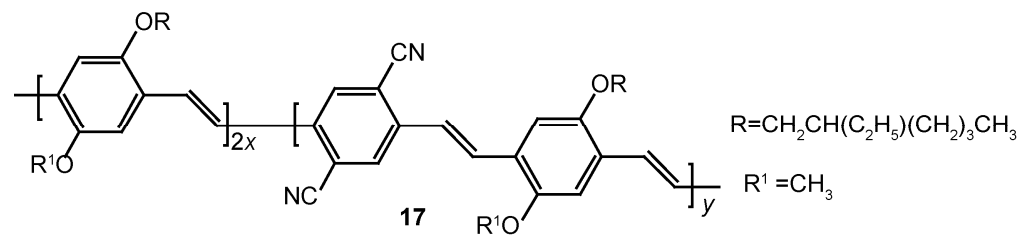

Figure 7

with an electron-defi- cient OXD side chain by mimicking the chemical structure of 2-(4-biphenylyl)-5-(4-tert-butylphenyl)-1,3,4-oxadiazole (PBD, Figure 8), which is widely used as ET material. Polymer $\mathbf{1 9}$ emits in the orange-yellow range because of its band gap of $2.36 \mathrm{eV}$. The HOMO and LUMO energy levels of $\mathbf{1 9}$ are -5.21 and $-2.56 \mathrm{eV}$, respectively, which indicates that $\mathbf{1 9}$ offers a similar electron-injection efficiency as PBD and better hole injection efficiency than PPV [25]. Huang et al. designed a facile synthetic route to a new conjugated polymer $\mathbf{2 0}$, in which a high-electron-affinity $\left(E_{\mathrm{a}}\right)$ aromatic OXD moiety is attached on the PPV backbone as a side chain. The HOMO and LUMO energy levels of polymer 20 are -5.47 and $-3.16 \mathrm{eV}$, respectively. This polymer offers a much better chargebalanced injection than 16. Attaching the high $E_{\mathrm{a}}$ OXD moiety onto the PPV backbone lowers both the HOMO and LUMO energy levels of the polymer, thus mitigating the problem of charge imbalance in OLEDs (Figure 9) [26].

In addition, there has been increasing interest in the silyl-substituted luminescent polymers, in which silyl moieties are introduced either as side chains or as a segment integrated into the polymer backbone. Incorporating silylene or oligosilylene segments into a polymer backbone has the
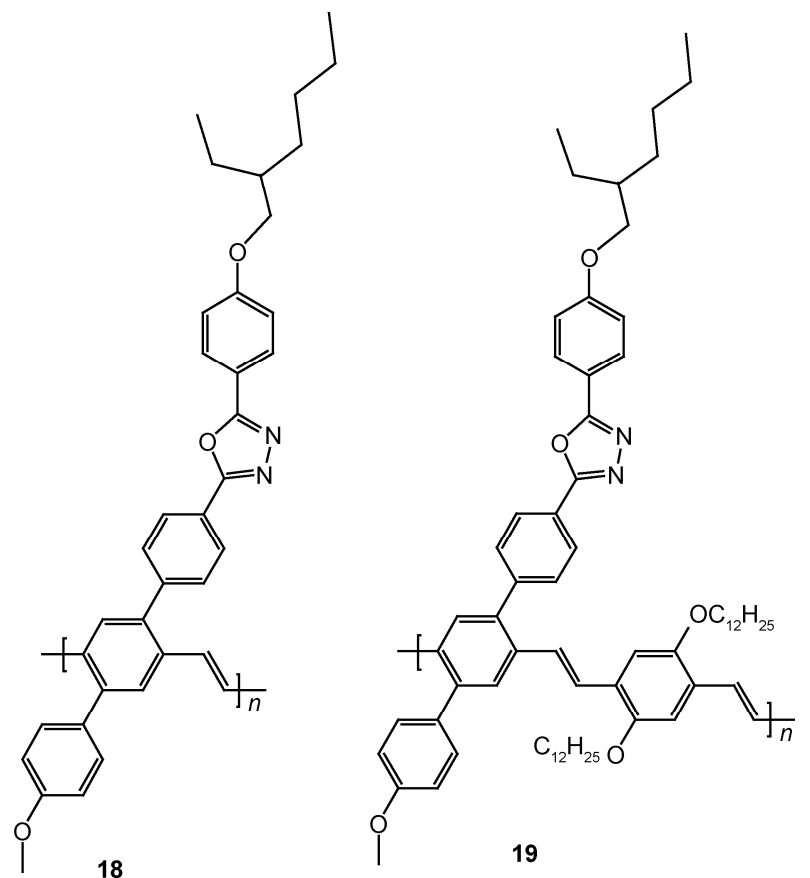

Figure 8

advantage of confining the electron delocalization over the polymer chain by alternating delocalized $\delta$ bonds and $\pi$ bonds to produce high-quantum-efficiency emission while also improving the flexibility of the resulting copolymers. Huang et al. synthesized PPV polymers 21-25, which contain silyl groups with chain lengths from $\mathrm{C} 1$ to $\mathrm{C} 18$ (Figure 10). The HOMO (LUMO) energy levels of polymers 22-25 are $-5.54(-2.60) \mathrm{eV},-5.52(-2.62) \mathrm{eV},-5.56(-2.58) \mathrm{eV}$, and $-5.62(-2.62) \mathrm{eV}$, respectively. Investigating the polymers with $\mathrm{CV}$ reveals that the side-chain length also plays an important role in the redox behavior of the polymers. Polymers with shorter side chains exhibit more reproducible CV scans and higher peak currents, which implies that the chemical-electrical stability and charge injection or transport for shorter-side-chain polymers are better than those for longer-side-chain polymers [27, 28]. Furthermore, the novel $p$ - $n$ diblock copolymer $\mathbf{1 4}$ demonstrates intrinsically balanced electron and hole injection and transport [29]. Incorporation of a bipyridine unit into a silicon-containing PPV-based polymer backbone can combine the merits of both components and yield a blue EL polymer 26 with high $E_{\text {a }}$ (Figure 11) [30].

The fluorene molecule is an isocyclic aromatic hydrocarbon composed of two benzene rings connected via a direct carbon-carbon bond and an adjacent methylene bridge. Owing to their wide energy gap from the backbone, highly efficient electroluminescence coupled with high chargecarrier mobility, and good processability, the fluorene molecule has emerged as an attractive class of conjugated materials. However, one drawback of fluorene-based blue EL materials is their long-wavelength emission, which occurs

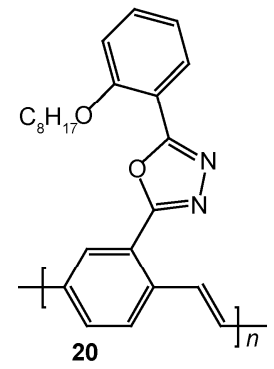

Figure 9

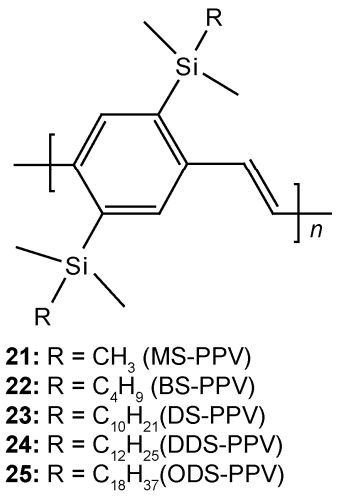

Figure 10 
after annealing the films in air or after operating the OLEDs for a long time. This emission strongly degrades the efficiency, color purity, and stability of OLEDs. Some researchers suggested that this long-wavelength emission was caused by excimer-related species [31], whereas others claimed that it was caused by the fluorenone [32,33]. Huang et al. designed a general synthetic strategy to prepare 2,7-dibromo-9-hetero- fluorenes, opening the door to new classes of inorganic and organometallic conjugated polymeric materials (e.g., 27) of $p-n$ diblock polyheterofluorenes (Figure 12) [34]. It is surprising that the oxidized phosphorus atom on phosphafluorene 28 and 29 significantly broadens the blue EL of polyfluorene (PF) into white emission with CIE coordinates of $(0.34,0.36)$ and a maximum luminescent brightness of $1423 \mathrm{~cd} / \mathrm{m}^{2}$, despite the actual incorporation ratio of phosphafluorene oxide being only $11 \%$ (Figure 13) [35].

\section{Adjusting the energy levels of organic conju- gated materials by synthesizing branched $p$-n diblock polymers}

In their investigation of linear polymers, Huang et al. also studied the feasibility of tuning the optoelectronic properties of branched $p-n$ diblock polymers. By introducing spiro-fluorene into the PF main chain, pure blue emission is obtained, which is attributed to the amorphous structure and weak interchain interaction of the polymer in the solid state as a result of the steric hindrance of the spiro-structure [36]. Huang et al. introduced the $p-n$ diblock skeleton into spiro-fluorene and synthesized the di-channel copolymer 30 based on fluorene and OXD (Figure 14). The HOMO energy level of the polymer is $-5.70 \mathrm{eV}$, which illustrates that the HOMO energy level is significantly altered by the

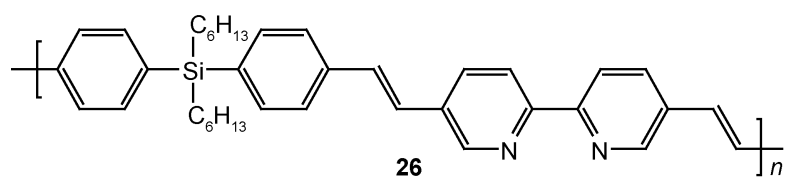

Figure 11
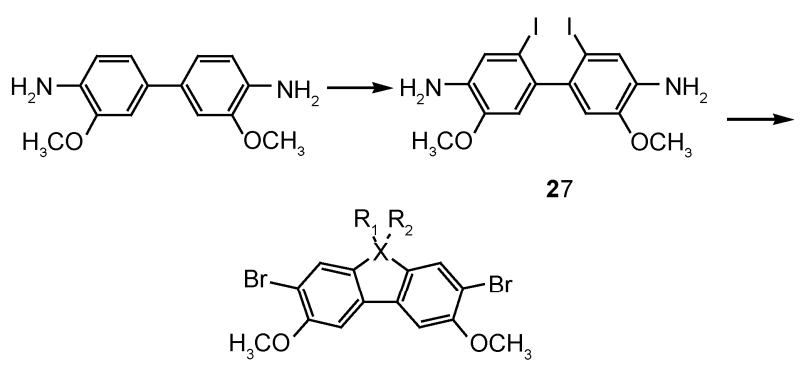

$X=\mathrm{Si}, \mathrm{Ge}, \mathrm{B}, \mathrm{P}, \mathrm{S}, \mathrm{Hg}, \mathrm{Ga}, \mathrm{In}$

Figure 12

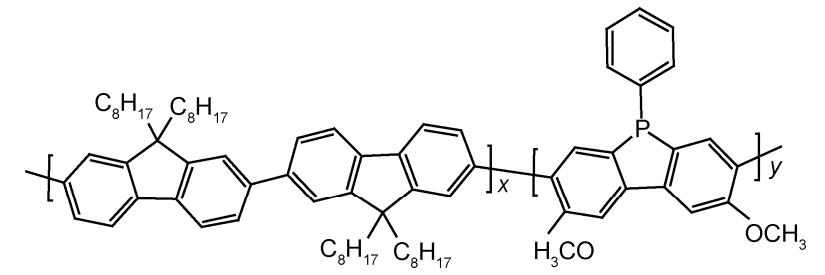

28

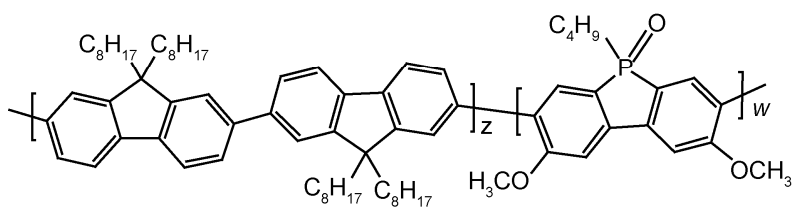

29

Figure 13

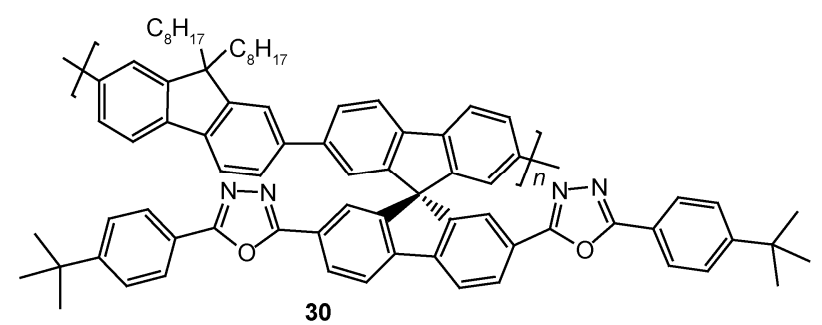

Figure 14

attachment of OXD branches onto the PF backbone through the spiro-bridge, and the $E_{\mathrm{a}}$ is significantly raised [37]. Huang et al. also find that hyperbranched OXDcontaining $p$ - $n$ diblock PFs (e.g., 31) exhibit very stable blue light emission even after being annealed at elevated temperatures in nitrogen or air (Figure 15) [38-40]. By introducing triazine into $\mathrm{PF}$, the electron injection and transport properties are also improved and green emission is suppressed, and OLEDs fabricated with polymer 32 show reasonably high external quantum efficiencies (Figure 16) [41]. Huang et al. synthesized a series of polymers, having sterically hindered side chains based on fluorene and pyridine derivatives $(\mathbf{3 3}, \mathbf{3 4}$, and $\mathbf{3 5}$, Figure 17$)$. The HOMO (LUMO) energy levels of polymer 33, 34, and $\mathbf{3 5}$ are $-5.75(-2.02) \mathrm{eV},-5.85(-2.41) \mathrm{eV}$, and $-5.75(-2.39)$ $\mathrm{eV}$, respectively, and the corresponding band gaps are 3.73 $\mathrm{eV}, 3.44 \mathrm{eV}$, and $3.36 \mathrm{eV}$. Although the absorption and PL-emission properties of these polymers are almost equivalent, their energy levels in thin solid films exhibit some differences that are correlated, to a large extent, with the branched molecular frameworks, which is consistent with theoretical calculations [42].

Controlled or living radical polymerization combines the virtues of living polymerization with the versatility and convenience of free radical polymerization [43]. The systems include atom-transfer radical polymerization, nitroxidemediated radical polymerization, and reversible addition-fragmentation-chain-transfer (RAFT) polymerization. Among all 


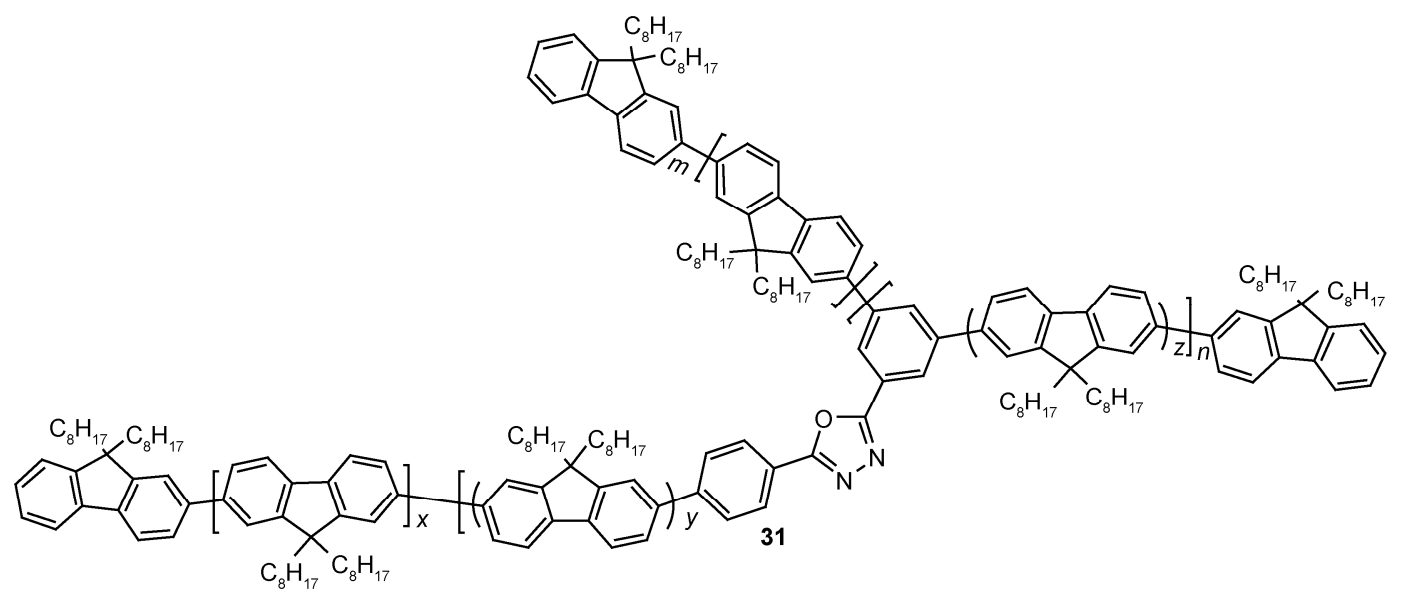

Figure 15

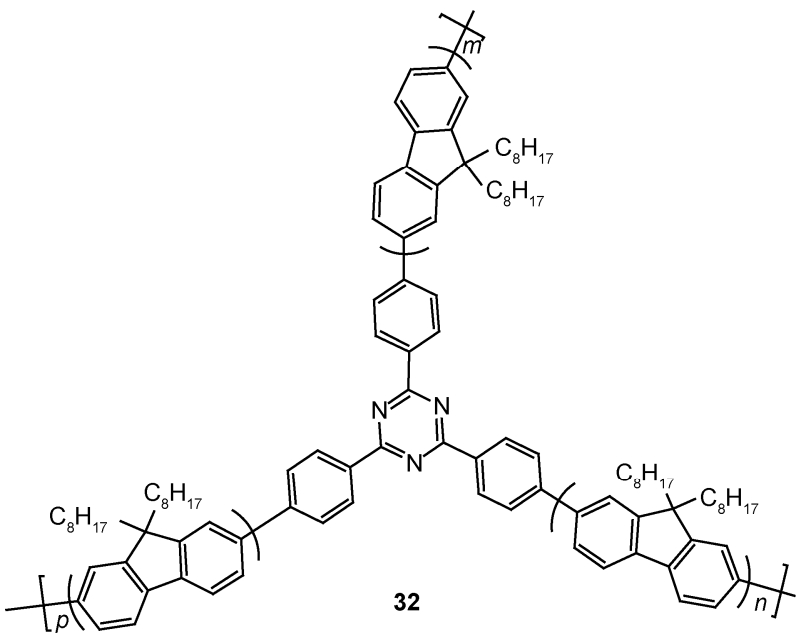

Figure 16

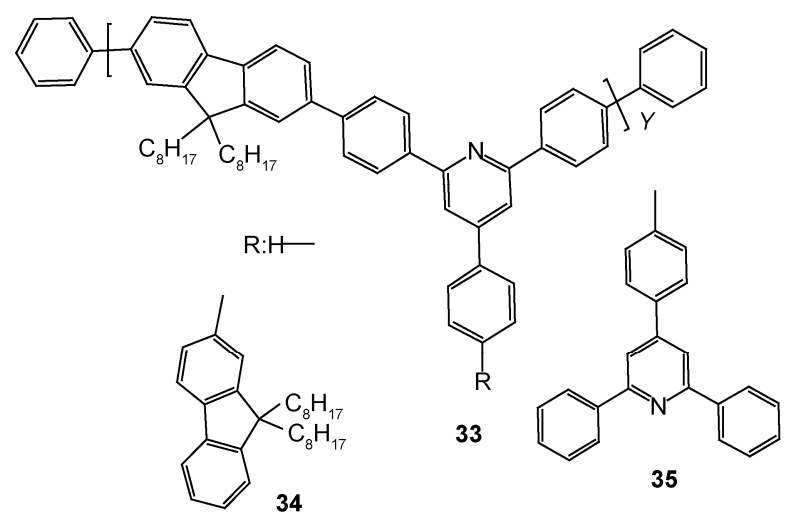

Figure 17

these polymerization techniques, the RAFT technique looks most promising because it is possible to control the homopolymerization of a great variety of monomers [44]. By using a RAFT procedure, Huang et al. synthesized functional polymers 36 and 37 with HT or ET ability using cumyl dithiobenzoate as a chain-transfer agent and 2,2'-
Azobis(2-methylpropionitrile) as an initiator (Figure 18). The HOMO (LUMO) energy levels of polymers $\mathbf{3 6}$ and $\mathbf{3 7}$ are $-5.45(-2.30) \mathrm{eV}$ and $-5.99(-2.34) \mathrm{eV}$, respectively, and the corresponding band gaps are $3.40 \mathrm{eV}$ and $3.65 \mathrm{eV}$, respectively. Good control of the RAFT polymerization is confirmed by the linear increase in molecular weight as a function of conversion [45,46].

In the past few years, organic phosphorescent lightemitting materials have attracted increasing attention because of their good performance and potential applications. Nearly $100 \%$ internal quantum efficiency can be achieved because the full utilization of singlet and triplet excitons is possible due to the strong spin-orbit mixing of heavy-metal
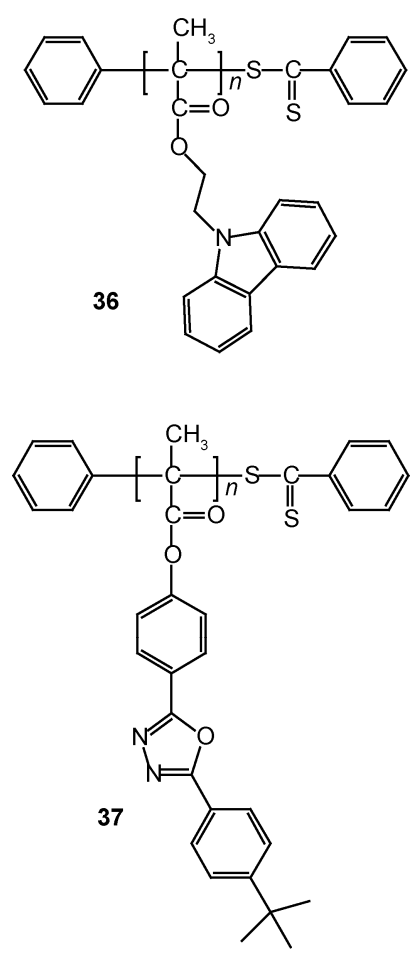

Figure 18 
ions in the complexes [47]. Huang et al. synthesized red-phosphorescent $\pi$-conjugated chelating polymers 38 that emit red light when the fluorene unit is copolymerized with the charged Ir complex (Figure 19) [48]. In the solid state, chelating polymers show almost complete energy transfer from the host fluorene segments to the guest Ir complexes when the feed ratio is only $2 \mathrm{~mol} \%$. The HOMO (LUMO) energy level of $\mathrm{PF}$ is $-5.8 \mathrm{eV}(-2.12) \mathrm{eV}$ [37]. The HOMO energy levels of $\pi$-conjugated chelating polymers are estimated to be about -5.76 to $-5.80 \mathrm{eV}$, and the LUMO energy levels are about -2.19 to $-2.25 \mathrm{eV}$. The HOMO energy levels of all chelating polymers are almost the same as that of PF, whereas the LUMO energy level is slightly lower than that of PF, which suggests that the chelating polymers have better electron injection and transport properties because of the incorporation of charged Ir complexes into the main polymer chain. Huang et al. also synthesized $\pi$-conjugated chelating polymer 39 with charged Ir complex units based on 1,10-phenanthroline in the backbones and saturated-red electrophosphorescence with CIE coordinates of $(0.61,0.30)$ was obtained [49,50]. Compared with those polymers containing charged Ir complexes, the addition of CBZ improves their hole-injection and chargeattraction ability as well as the host-guest energy-transfer efficiency (Figure 20) [51].

\section{Adjusting the energy levels of organic conju- gated materials by synthesizing $p$ - $n$ diblock oli- gomers}

There has been extensively investigation of $\pi$-conjugated polymers over the past decades because of their potential applications in semiconductor devices. Most of these applications would benefit from a full understanding of charge transport (CT) properties, which depend on the morphology of thin films and their electronic structure. As mentioned above, the $p$ - $n$ diblock concept developed by Huang et al. has led to the synthesis of several series of $p$ - $n$ diblock conjugated polymers. By varying the length of the $p$ and $n$ segments of the block polymer chain, it is possible to tune the HOMO and LUMO energy levels, thereby the emissive wavelength, and these polymers exhibit improved PL and EL properties. However, the improvement is still, to some degree, less than expected. The subpar improvement may be attributed to the alternating distribution of the $p$ and $n$ segments in the polymer chain. The electron-deficient unit inserted into the $p$-type polymer chain will partially act as a hole-blocking unit because of its high $E_{\mathrm{a}}$. Conversely, the HT unit will lower the electron mobility. Furthermore, deep electron traps may occur in polymeric systems as a result of chain entanglement or structural defects. The above-mentioned drawbacks can be resolved by block oligomers that have separate $p$-type and $n$-type blocks [52]. The $p$-n diblock oligomers, which are analogous to the threedimensional semiconductor $p-n$ junction, combines easy

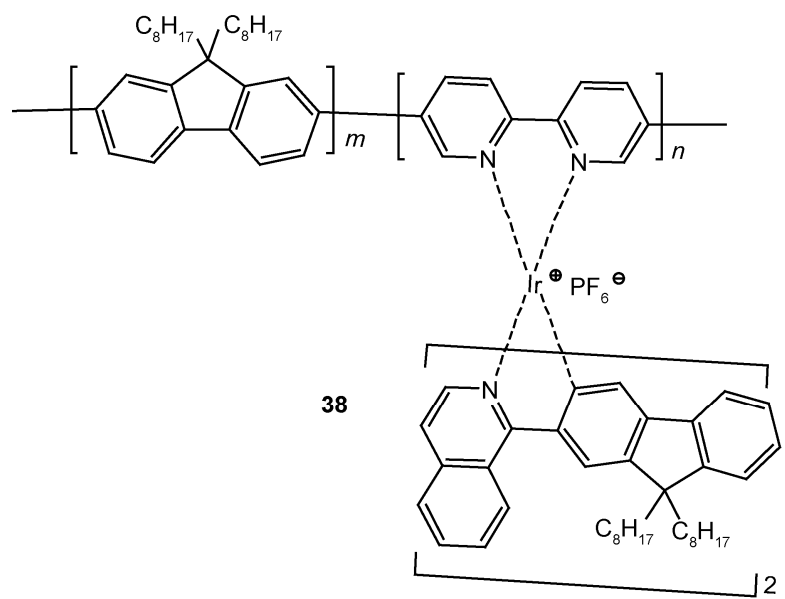

Figure 19

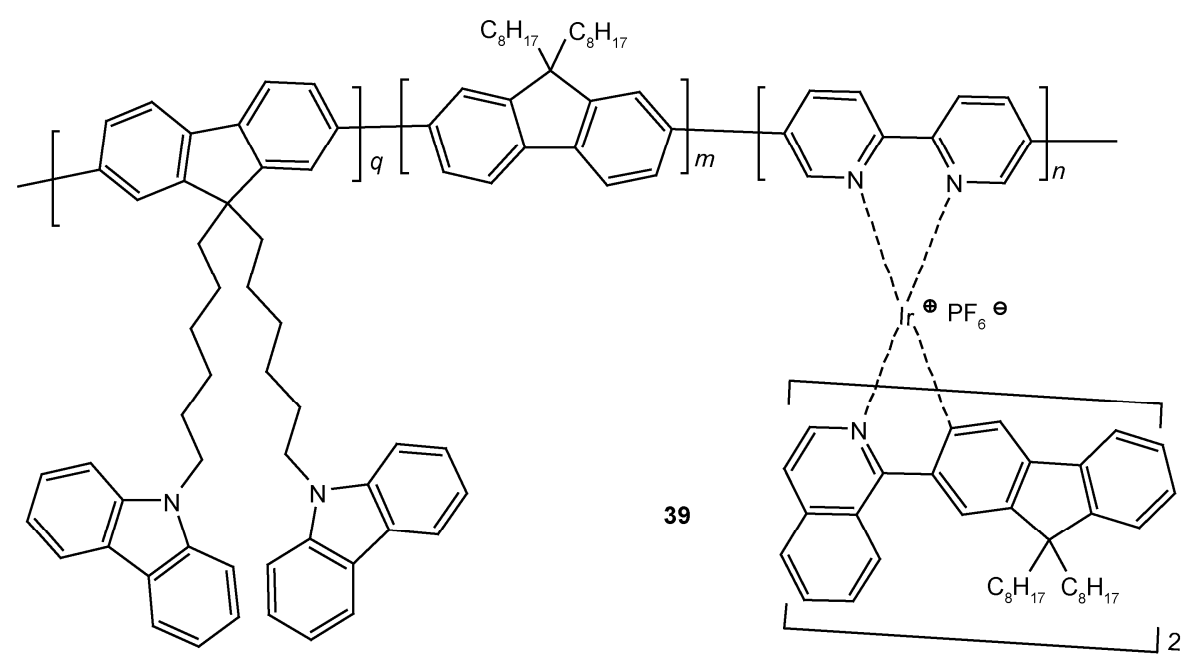

Figure 20 
charge injection with current rectification properties. Additionally, in comparison with conjugated copolymers, the monodisperse conjugated oligomers possess well-defined conjugation lengths and structures, which characterized by structural uniformity and ease of purification and characterization.

To investigate these materials, Huang et al. synthesized novel $p-n$ diblock and triblock oligomers 40-44, which consist of THP as the $p$-type unit and OXD as the $n$-type unit (Figure 21). The HOMO (LUMO) energy levels of 40-44 in solution are $-5.37(-2.37) \mathrm{eV},-5.39(-2.72) \mathrm{eV}$, $-5.15(-2.70) \mathrm{eV},-5.30(-2.40) \mathrm{eV}$, and $-5.38(-2.60) \mathrm{eV}$, respectively. By changing the molecular regiochemistry and the number of THP and OXD units, it is possible to modu- late the optoelectronic properties of the oligomers [53]. Huang et al. also synthesized the two fluorene and trifluoromethyl end-capped linear phenylene/OXD oligomers 45 and 46 (Figure 22), which were incorporated as the ET/ hole-blocking material in an OLED that displayed highly efficient red electroluminescence [54].

The organic conjugation length and carrier mobility of the linear oligomers are combined in one dimension, which makes it difficult to evaluate separately the actual contribution of two functions [55]. To resolve this matter, Huang et al. synthesized the cruciform $p-n$ diblock conjugated oligomers 47-51 using benzene as the core, a single $p$-type oligofluorene as the hole channel, and the $n$-type $\operatorname{di}(1,3,4-$

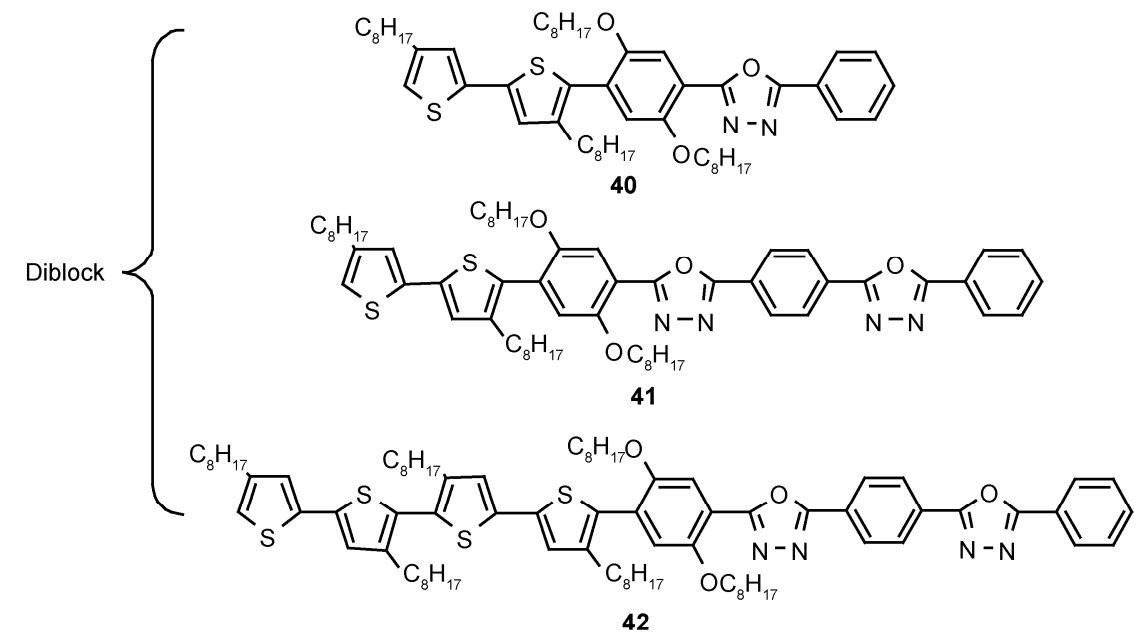

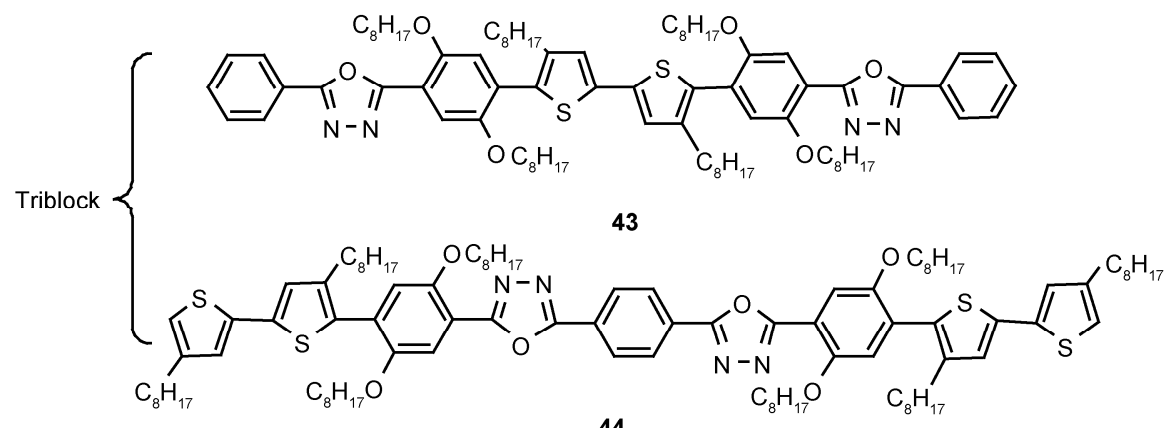

Figure 21<smiles>Fc1ccc(-c2ccc(-c3ccc(-c4nnc(-c5ccc(-c6ccc(F)cc6)cc5)o4)cc3)cc2)cc1</smiles>

Figure 22 oxadiazole) phenylene branch as the electron channel for OLEDs (Figure 23). It is useful to correlate the molecular structure with device properties upon changing the composition, chemical structure, and proportion of $p$-type or $n$-type branches. The HOMO (LUMO) energy levels of oligomers 45, 48, and 49 in solution are $-5.79(-2.64) \mathrm{eV}$, $-5.60(-2.67) \mathrm{eV}$, and $-5.54(-2.62) \mathrm{eV}$, respectively. The maximum luminance of oligomer 49 in a device is 2622 $\mathrm{cd} / \mathrm{m}^{2}$ at $16.5 \mathrm{~V}$ and its luminance efficiency is $1.6 \mathrm{~cd} / \mathrm{A}$, which is much higher than that of polymer 52. The lower turn-on voltage and higher maximum brightness are due to better charge injection and transport and efficient charge 

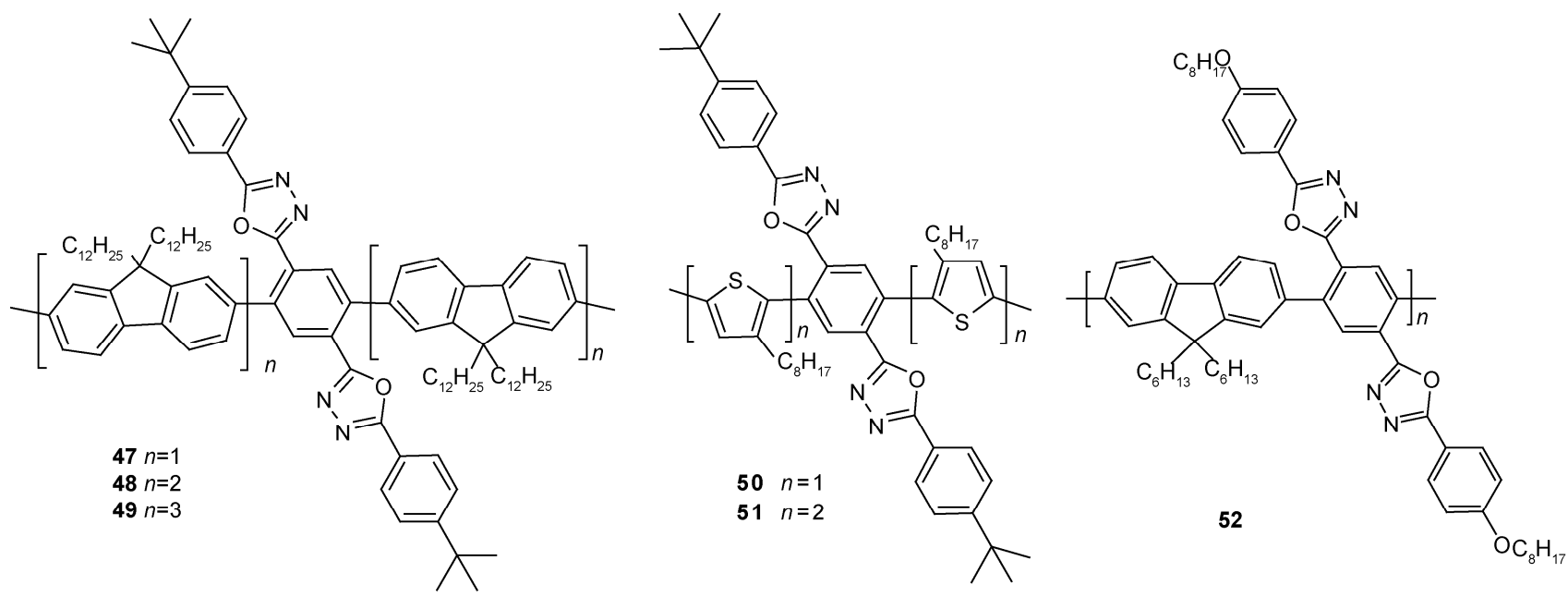

Figure 23

recombination, which result from the two independent channels in the cruciform. The oligofluorene branch acts as a HT channel, whereas the di(1,3,4-oxadiazole)phenylene branch acts as an ET channel [56,57]. These oligomers further enriched the category of the di-channel copolymers. To investigate the fine optoelectronic difference of the oligomers with or without peripheral fluorene moieties, Huang et al. prepared two dumbbell-shaped oligomers $\mathbf{5 3}$ and $\mathbf{5 4}$ (Figure 24). The HOMO (LUMO) energy levels of $\mathbf{5 3}$ and 54 in solution are $-5.55(-2.42) \mathrm{eV}$ and $-5.66(-2.43) \mathrm{eV}$, respectively, and the corresponding band gaps are $3.17 \mathrm{eV}$ and $3.24 \mathrm{eV}$. Theoretical calculations that reproduce the observed band gaps of these oligomers further our understanding of the different molecular orbital contours of the HOMO energy levels (Figure 25) [58]. The ultraviolet-visible (UV-Vis) absorption and PL properties are rarely affected by molecular architecture, whereas the absolute PL quantum efficiency of films and the energy band gaps derived from $\mathrm{CV}$ in solution are strongly correlated with the molecular framework.

For decades, lanthanide complexes have been studied as emitters for OLEDs because they have nearly monochromic emission, chemical environmental stability, and almost $100 \%$ theoretical internal device quantum efficiency. However, for multiple reasons, the EL performance of these lanthanide materials is still much lower than expected. The

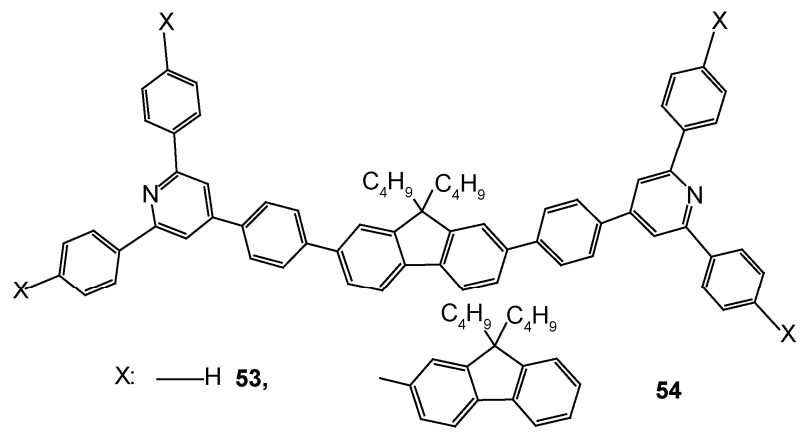

Figure 24 unbalanced hole and electron injection and transport is undoubtedly one of the most important factors explaining this deception. In most lanthanide complexes, the materials are better electron transporter than hole transporters [59].

Therefore, much work has focused on improving the HT ability of lanthanide complexes through by modifying the HT moieties [60]. Huang et al. synthesized three $\mathrm{Eu}^{\text {III }}$ complexes 55, 56, and $\mathbf{5 7}$ by combining the functional singlecoordinate phosphine oxide ligands with the most compact structure (Figure 26). The HOMO (LUMO) energy levels of oligomers 55, 56, and 57 are $-5.24(-3.00) \mathrm{eV},-5.29$ $(-3.08) \mathrm{eV}$, and $-5.69(-3.03) \mathrm{eV}$, respectively. The results show that, by taking advantage of the modification inertia of the phosphine oxide ligands, the direct introduction of the HT groups as chromophores results in the most compact structure and mezzo $S_{1}$ and $T_{1}$ energy levels, which improves the intramolecular energy transfer in the $\mathrm{Eu}^{\mathrm{III}}$ complexes and is consistent with theoretical calculations. Both four-layer devices based on pure $\mathbf{5 5}$ and $\mathbf{5 6}$ have a maximum brightness of more than $1000 \mathrm{~cd} / \mathrm{m}^{2}$, turn-on voltages below $5 \mathrm{~V}$, maximum external quantum yields over $3 \%$, and excellent spectral stability [61]. Two CBZ-based phosphine oxide ligands with bipolar structures and their corresponding Eu ${ }^{\text {III }}$ complexes $\mathbf{5 7}$ and $\mathbf{5 8}$ are chosen to investigate the effects of the different structures of the ligands on the electrochemical, PL, and EL properties of the complexes [62]. The adaptability of the $p-\pi-n$ systems, especially for coordination, is superior to that of $p-n$ systems, which facilitates the modification of the complexes by purposely designing multifunctional ligands. Compound $\mathbf{5 7}$ seems favorable as the most efficient EL Eu ${ }^{\text {III }}$ complex with greater brightness, higher efficiencies, and a more stable EL spectrum than $\mathbf{5 8 .}$

\section{Theoretical calculations of the energy levels of organic $p-n$ diblock conjugated materials}

The $p$ - $n$ diblock molecules fabricated via the molecular 


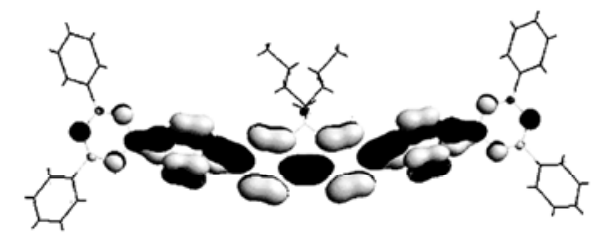

53-LUMO

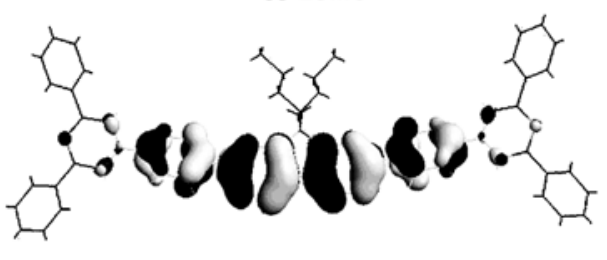

53-HOMO

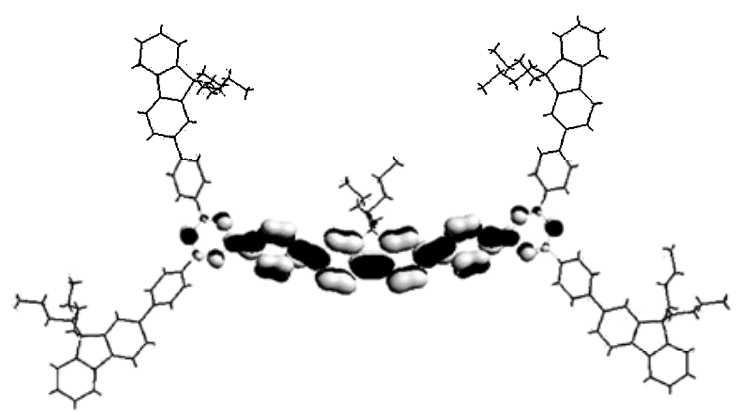

54-LUMO

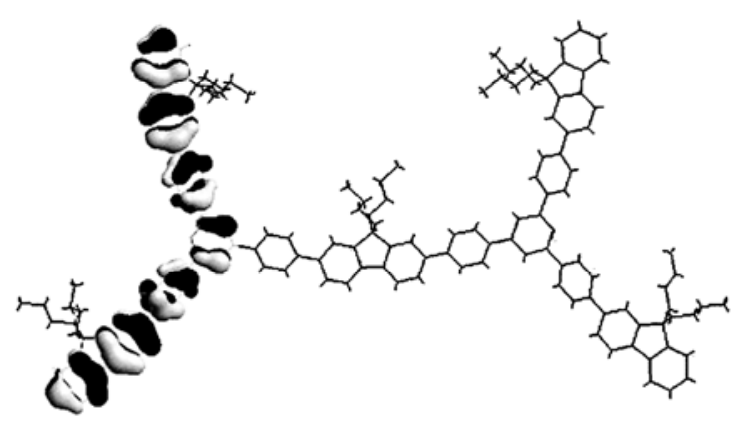

54-HOMO

Figure 25 Molecular orbital contours of the HOMO and LUMO for $\mathbf{5 3}$ and 54 from AM1 calculations [61].

design and synthesis proposed by Huang et al. can be used as molecular diodes, and the electronic and optoelectronic properties related to the HOMO and LUMO energy levels of the polymers can be tailored by varying the conjugation length, the regiochemistry of the oligothiophene, or the other blocks in the repeating unit. To better understand how to tune $p$ - $n$ diblock molecules, they also performed quantum chemical calculations on the oligomer representatives with the same $p-n$ diblock structure [63-65]. Recently, Huang et al. performed semiempirical calculations to investigate the tunable behavior of the $p$ - $n$ diblock copolymers 59-65 with repeating units constructed by oligothiophenes as $p$-doped blocks and 1,4-bis(oxadiazolyl) benzene as $n$-doped blocks (Figure 27). The calculated evolution of the HOMO and LUMO energy levels of the model oligomers agree well with the experimental oxidation and reduction potentials of the corresponding polymers, and the backbone modification of the $p$ - $n$ diblock copolymer, which implies changing the number of THP unit in the $p-n$ diblock copolymer, greatly modifies the optoelectronic properties of the polymer [66-69].

Apart from the backbone modification of the $p$ - $n$ diblock molecules mentioned above, a lot of approaches have been proposed to adjust the optoelectronic properties of organic conjugated materials. PPV derivatives have received a considerable amount of attention because of their applications in OLED. The most challenging task in this field is to optimize the photophysical behavior of PPVs to achieve high quantum efficiencies for both photo- and electroluminescence. Fluorinated oligophenylenevinylenes having different alkyl or alkoxy groups on the aryl ring can be useful in understanding the relationship between luminescence and structure. Fluorine is a strong electron-withdraw- ing group, while methyl and methoxy are donor (D) groups. Therefore, compounds having these functional groups can help us to understand the behavior of oligophenylenevinylenes in the excited state.

Neckers et al. investigated the effect of aromatic ring substitution on the optoelectronic properties, emission dynamics, and solid-state behavior of the fluorinated oligophenylenevinylenes 66 and 67 (Figure 28) [70]. Fluorinated oligo ( $p$-phenylenevinylene)s with alkoxy substituents open new directions for the development of devices and fluorescent probes. Generally, ET organic semiconductors are relatively rare, but of great interest for organic electronics. The most prominent examples of thin-film-transistor (TFT) $n$-type materials have been obtained by modifying known $p$-type cores with strongly electronegative fluoro/luoroalkyl substituents. However, despite these advances, factors underlying fluoro stabilization of the injected electrons are not fully understood, which has often been attributed to a reduction in the LUMO energy level. Of the known THP- based semiconductors, the vast majority exhibit HT TFT activity. However, $n$-type THP-based oligomers and polymers are extremely useful because the stability and ready functionalizability of the oligothiophene cores.

Marks et al. synthesized a new polythiophenefluoroarene family of conducting polymers (68-71), which allows the direct scrutiny of regiochemical effects on crystal structure, orbital energies, and CT [71] (Figure 29). The HOMO (LUMO) energy levels of 68, 69, 70, and 71 in solution are $-5.20(-2.65) \mathrm{eV},-5.48(-2.85) \mathrm{eV},-5.64(-2.82) \mathrm{eV}$, and $-5.81(-2.81) \mathrm{eV}$, respectively, whereas those in thin solid film are $-4.78(-2.36) \mathrm{eV},-5.27(-2.69) \mathrm{eV},-5.32(-2.67)$ $\mathrm{eV}$, and $-5.40(-2.53) \mathrm{eV}$, respectively. A new family of closely packed polythiophene-fluoroarene conductors is obtained, and its carrier mobility can be drastically modulated by changing the molecular regiochemistry.

Red OLEDs are an indispensable component for fullcolor displays. However, red-light-emitting materials with 

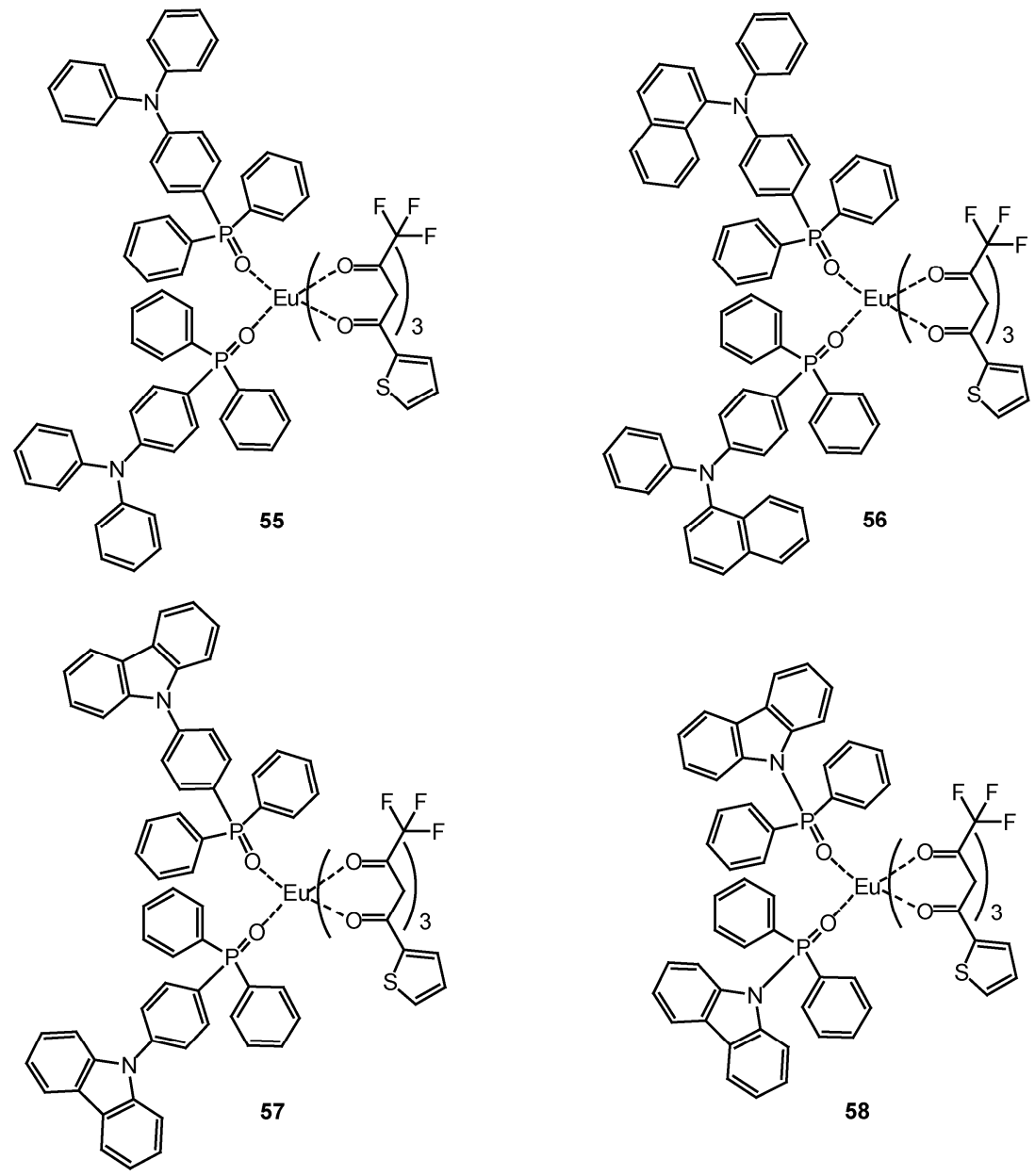

Figure 26

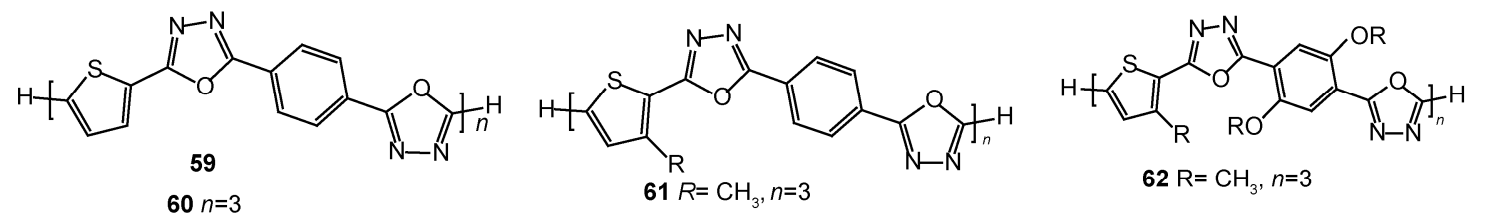<smiles>c1csc(-c2ccc(-c3ccc(-c4nnc(-c5ccc(-c6nn[14cH]o6)cc5)o4)s3)s2)c1</smiles><smiles></smiles>

Figure 27

high brightness and pure color fidelity are scarce compared with other light-emitting materials. The reason for this is that organic red-light-emitting materials are usually molecules that have either extended $\pi$-conjugated systems or bear $p-n$ polar subsistents, which tend to crystallize in the solid state [73]. Thus, most of the materials developed to date suffer from severe concentration quenching. Consequently, most red OLEDs use a dopant system. Liu et al. synthesized two red-light-emitting compounds $\mathbf{7 2}$ and $\mathbf{7 3}$, which have obvious intramolecular charge-transfer charac- teristics (Figure 30). Although the molecular structure has minor effects on the energy level of the compound, the HOMO (-5.3 eV) and LUMO (-3.4 eV) energy levels can enhance electron injection for potential use in ET. Three types of multilayered OLEDs were made using these materials as host-emitting and ET layers. Under ambient conditions, a layer of 73 has a maximum luminance of $536 \mathrm{~cd} / \mathrm{m}^{2}$ and a maximum external quantum efficiency of $0.7 \%$ [73]. However, most blue-emitting materials exhibit large band gaps and low $\mathrm{E}_{\mathrm{a}} \mathrm{s}$, leading to inefficient electron injection 


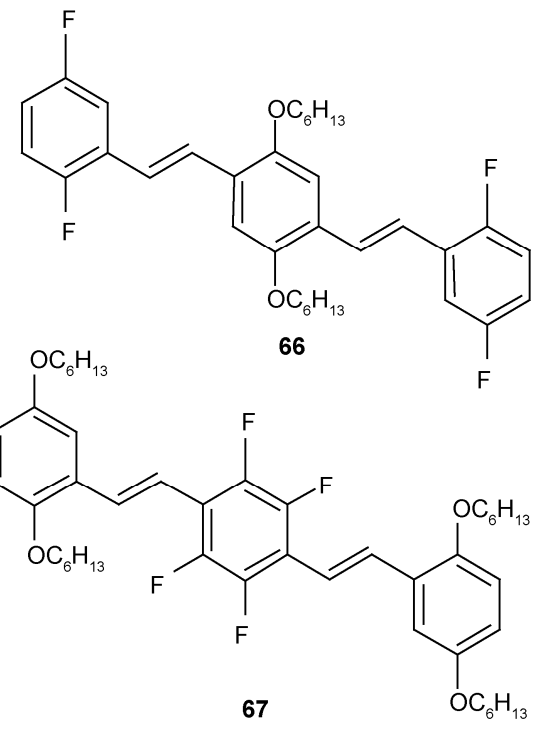

Figure 28

into blue emitters. Therefore, it is necessary to synthesize blue-emitting materials with high $E_{\mathrm{a}}$ values to facilitate electron injection and transport [74]. Liu et al. [75] have synthesized compounds $\mathbf{7 4}$ and $\mathbf{7 5}$ (Figure 31), which exhibit remarkably high thermal stabilities. The HOMO (LUMO) energy levels of $\mathbf{7 4}$ and $\mathbf{7 5}$ in solution are -6.2 $(-3.3) \mathrm{eV}$ and $-5.7(-2.6) \mathrm{eV}$, respectively. The relatively lower LUMO energy level of $\mathbf{7 4}$ makes it a good ET material. Using $\mathbf{7 5}$ as an exciplex-eliminating layer and $\mathbf{7 4}$ as the emissive layer, they fabricated a high-efficiency blue OLED with a maximum luminance of $6230 \mathrm{~cd} / \mathrm{m}^{2}$ and a maximum current efficiency of $5.2 \mathrm{~cd} / \mathrm{A}$ [76].

The three most successful approaches to reducing the band gap in organic compounds are: (1) extending the $\pi$-conjugation, (2) tuning bond-length alternation in the

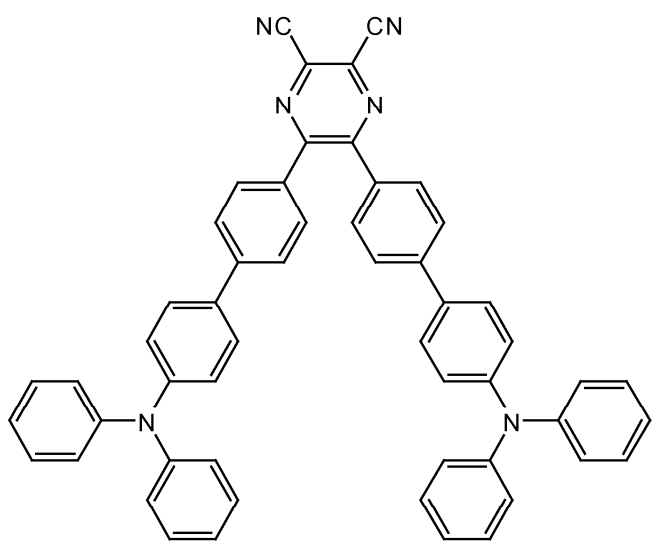

72<smiles>c1csc(-c2ccc(-c3ccc(-c4ccc(-c5ccc(-c6cccs6)s5)s4)s3)s2)c1</smiles>

68<smiles>Fc1c(F)c(F)c(-c2ccc(-c3ccc(-c4ccc(-c5ccc(-c6c(F)c(F)c(F)c(F)c6F)s5)s4)s3)s2)c(F)c1F</smiles><smiles>Fc1c(F)c(-c2ccc(-c3ccc(-c4c(F)c(F)c(-c5cccs5)c(F)c4F)s3)s2)c(F)c(F)c1-c1cccs1</smiles><smiles>Fc1c(F)c(-c2c(F)c(F)c(-c3ccc(-c4cccs4)s3)c(F)c2F)c(F)c(F)c1-c1ccc(-c2cccs2)s1</smiles>

Figure 29

$\pi$-systems by stabilizing a quinoidal character, and (3) introducing appropriate D and acceptor (A) units. Among many low-band-gap organic compounds, the $p$ - $n$ diblock type of chromophores are promising candidates for near-infrared light-emitting materials, because their bandgap levels can be readily tuned by adjusting the strength

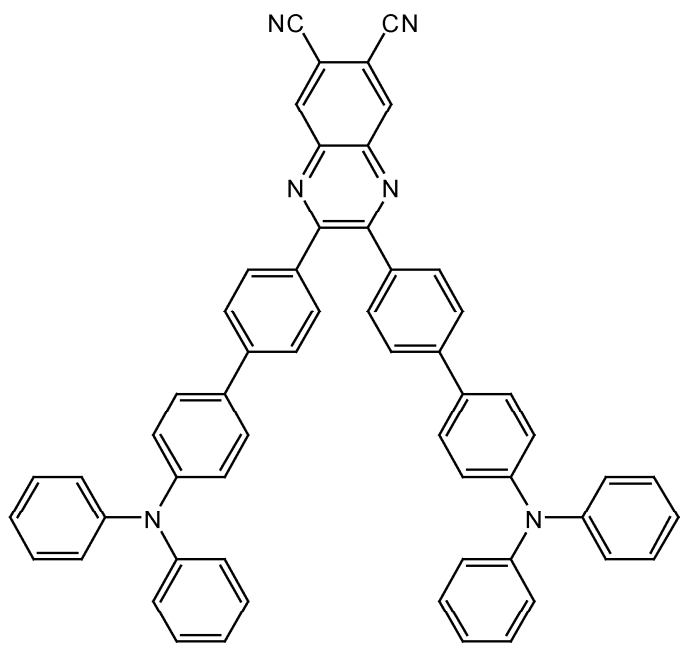

73

Figure 30 


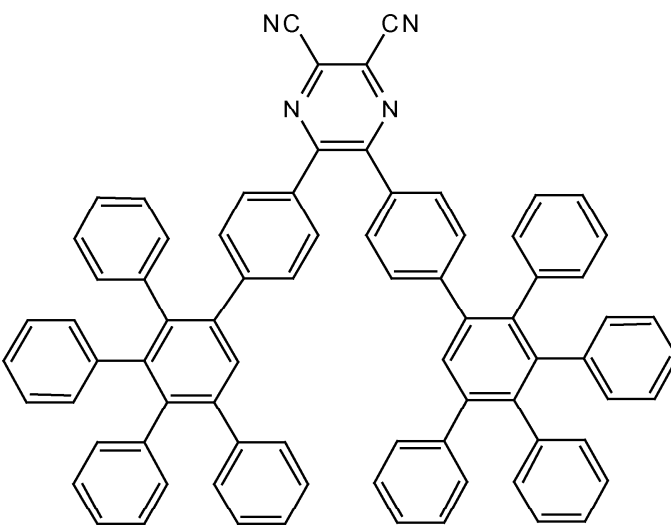

74

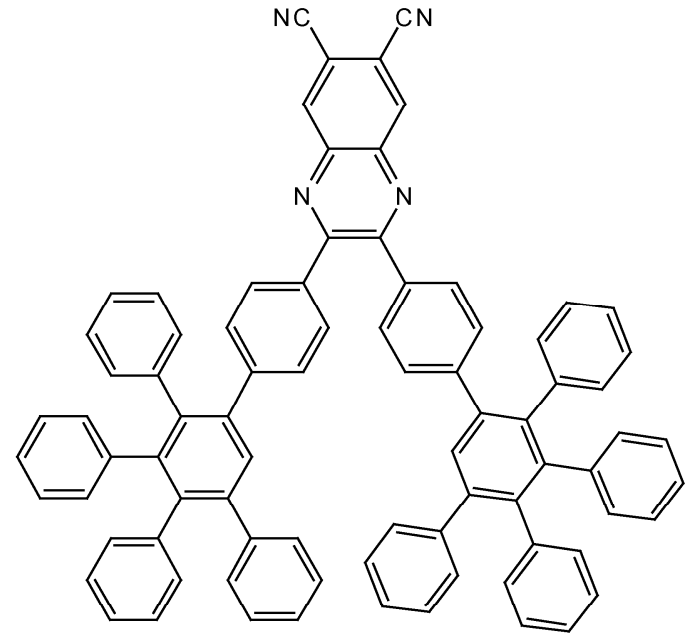

75

Figure 31

of the $\mathrm{D}$ and $\mathrm{A}$ and can reach below $1.24 \mathrm{eV}$ (or above 1000 $\mathrm{nm})$. Wang et al. report the synthesis of a series of structurally simple D- $\pi$-A- $\pi$-D near-infrared chromophores 76-79, (Figure 32), and demonstrate nondoped near-infrared OLEDs with a single emission peak centered at $1000 \mathrm{~nm}<$ $\lambda<1220 \mathrm{~nm}$. A high external quantum efficiency of $0.28 \%$ at the emission wavelength of $1080 \mathrm{~nm}$, with a maximum radiance of $200 \mathrm{~mW} \mathrm{Sr}^{-1} \mathrm{~m}^{-2}$ is achieved [77].

The use of electrogenerated films offers the advantage of a one-step polymer synthesis that allows fine control over the film thickness. Wong et al. synthesized a 9,9-spirobifluorene-cored $p-n$ bichromophore system in which the electron-donating moieties were triphenylamine (TPA) and
CBZ groups and the electron-withdrawing moieties were OXD groups. The electron-deficient OXD groups efficiently blocked the radical cation delocalization between the two terminal TPA groups, rendering the electropolymerization of the TPA groups feasible (Figure 33). The polymer films made of $\mathbf{8 0}$ and $\mathbf{8 1}$ exhibited reversible electrochemical oxidation, accompanied by strong color changes with high coloration efficiency and contrast ratio that could be switched through potential modulation [78]. Electrodeposition of spirobifluorene $p$ - $n$ diblock systems is a versatile tool for tuning the electrode surface work function [79]. Jenekhe et al. designed a new synthetic route to soluble polyquinolines 82-87 with tunable photophysical, redox, and<smiles></smiles><smiles>Cc1ccc(N(c2ccccc2)c2ccc(-c3c4c(c(-c5ccc(N(c6ccccc6)c6ccc(C)cc6)cc5)c5nsnc35)N=S=N4)cc2)cc1</smiles>

76

$$
77
$$<smiles>c1ccc(N(c2ccccc2)c2ccc(-c3c4c(c(-c5ccc(N(c6ccccc6)c6cccc7ccccc67)cc5)c5nsnc35)N=S=N4)cc2)cc1</smiles><smiles>c1ccc(N(c2ccccc2)c2ccc(-c3c4c(c(-c5ccc(N(c6ccccc6)c6ccccc6)s5)c5nsnc35)N=S=N4)s2)cc1</smiles>
79 


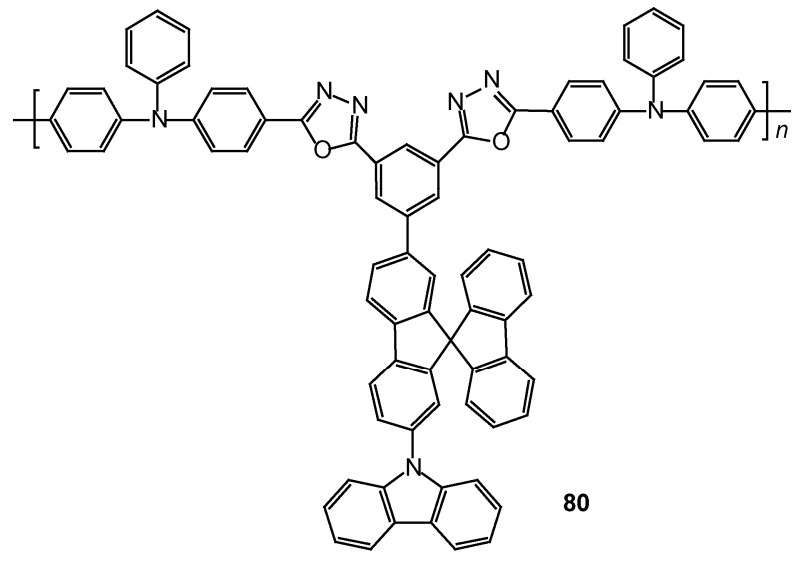

Figure 33

EL properties (Figure 34). The HOMO (LUMO) energy levels of thin films made from polymers 83-87 are -5.96 $(-1.56) \mathrm{eV},-5.61(-1.21) \mathrm{eV},-5.43(-1.03) \mathrm{eV},-5.30$ $(-0.90) \mathrm{eV}$, and $-5.17(-0.77) \mathrm{eV}$, respectively, while their PL emission maxima are at 493, 477, 524, 615, 646, and $576 \mathrm{~nm}$, respectively. Blue-green, green, yellow, orange and red EL emissions are available with moderate brightness and efficiency. By varying the arylene linkage in polymers from $p$-phenylene to bis(thienyl)vinylene, the optical band gap, redox properties and EL color can be tuned over a wide range. The new polyquinolines are also useful as ET materials in OLEDs $[80,81]$. Karabunarliev et al. presented a comparative theoretical study of the EL yield in $p$ - $n$ diblock polymers, and derived the rates of the spontaneous one-photon and one-phonon processes within a diabatic model adjusted to the electron-vibrational spectroscopy of PPV [82].

In recent years, conjugated polymers containing metal complexes, especially phosphorescent heavy-metal complexes, have attracted increasing attention. Energy can be efficiently transferred from the conjugated main-chain to metal complexes in the polymer main chain or side chains, which is very effective for producing long-wavelengthemission materials [83]. Iridium complexes are of paramount importance in this field because of their high PL quantum yields and appropriate exciton lifetimes. Unfortunately, either quenching of the luminescence caused by intermolecular interactions or poor carrier mobility has prevented these materials from being used in an undiluted form as emissive layers. Therefore, in many cases, a doping technique must be employed to fabricate high-performance devices. Chen et al. [84] reported new EL polymers that consists of grafting high-efficiency cyclometalated iridium complexes as phosphorescent dopants and CT moieties onto the alky side chains of fully conjugated polymers for use in single-layer-single-polymer OLEDs (Figure 35). The polymer $\mathbf{8 8}$ involves $\mathrm{PF}$ as the base conjugated polymer, $\mathrm{CBZ}$ as the CT moiety, and a source for green emission by forming an electroplex with the PF main chain. Devices prepared

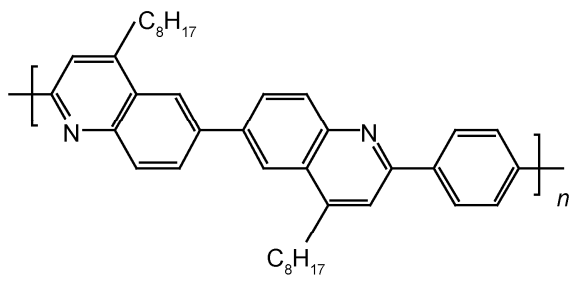

81

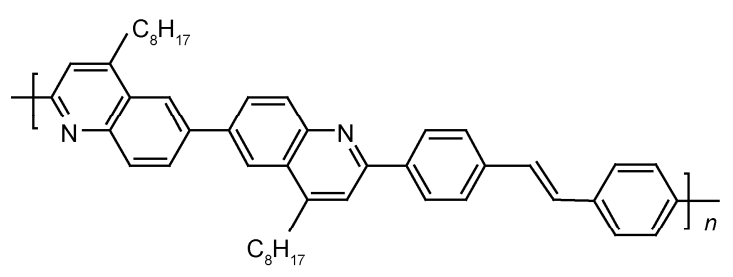

83

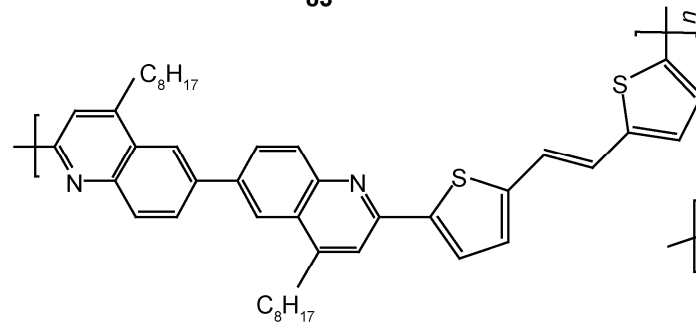

85

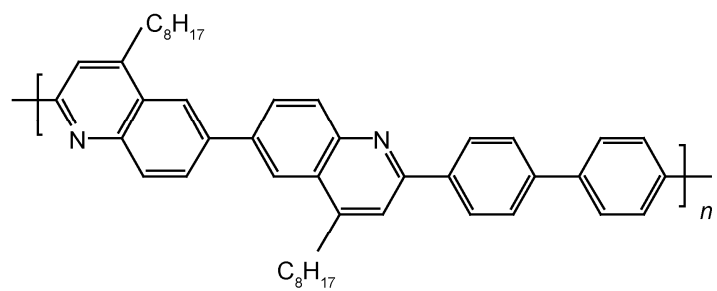

82

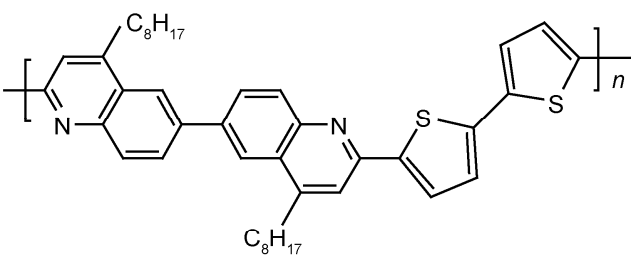

84

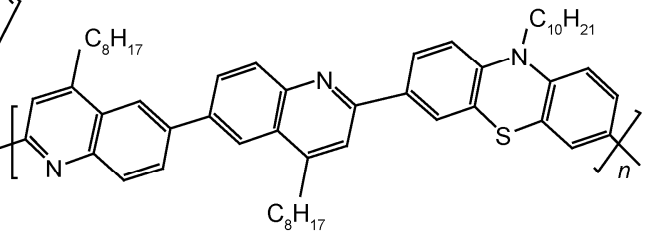

86

Figure 34 
from this material emit red light with a high efficiency of $2.8 \mathrm{~cd} / \mathrm{A}$ at $7 \mathrm{~V}$ and $65 \mathrm{~cd} / \mathrm{m}^{2}$. Because it is comparable with that of the same Ir-complex-based OLED, this polymer offers broad-band emission that contains blue, green, and red peaks. Dispersion of the Ir complex in a host matrix not only separates the phosphors and avoids self-quenching, but also contributes to CT.

White organic light-emitting devices (WOLEDs) have also attracted significant interest of late because of their strong potential for various lighting applications. An ideal white emission should contain three primary colors and cover the entire visible range from 400 to $700 \mathrm{~nm}$. To span the entire visible spectrum, most WOLEDs reported to date rely on the combinations of several organic components that emit different colors. Because they offer easy fabrication and low-cost processing, single-component white-light EL materials are highly sought for potential applications in OLEDs, flat panel displays and electronic paper displays [85].

Yang et al. reported a new strategy to produce efficient white-light emission from a single $p$ - $n$ diblock polymer, which consists of both PL- and phosphorescence-emitting species. By introducing a small concentration of benzothiadiazole (BT) units into the PF backbone and attaching a small number of iridium complexes onto the side chain, white-light emission is obtained based on three individual emission species. Large-band-gap fluorene is used as the host for the blue-light-emitting species, and BT is used as the green-light emitter. An iridium complex is used as the red-light-emis- sion triplet species because of its high PL quantum efficiency that results from strong spin-orbit coupling with the heavy-metal ions. By adjusting the concentration of BT or of the iridium complex in polymer 89, the simultaneous emission of the three species can be obtained and combined to form white light (Figure 36). Some devices emit white cd/A at a current density of $2.2 \mathrm{~mA} / \mathrm{cm}^{2}$. A maximum luminance of $10110 \mathrm{~cd} / \mathrm{m}^{2}$ is achieved at a current density light with CIE coordinates of $(0.34,0.33)$ and $(0.32,0.33)$, and some exhibit a maximum luminance efficiency of 6.1 of $345 \mathrm{~mA} / \mathrm{cm}^{2}$. The white-light emission of devices made from the copolymers is stable over the entire

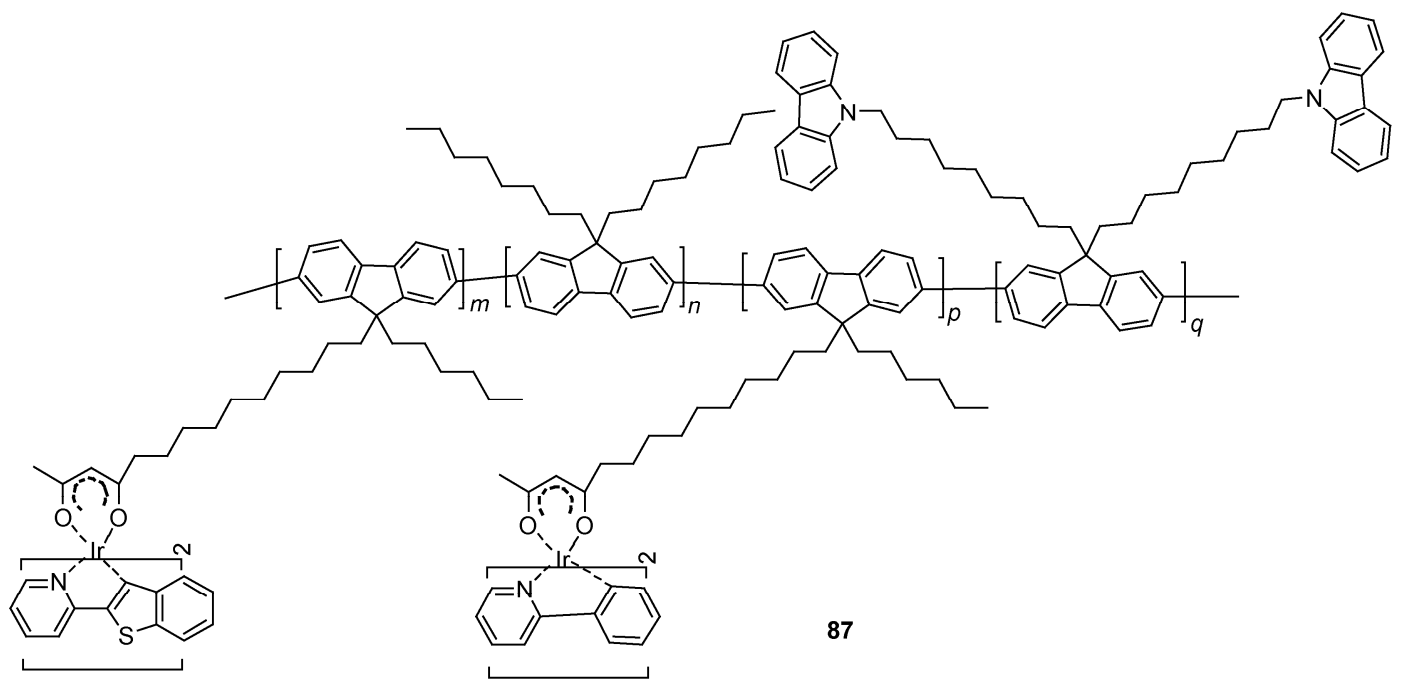

Figure 35
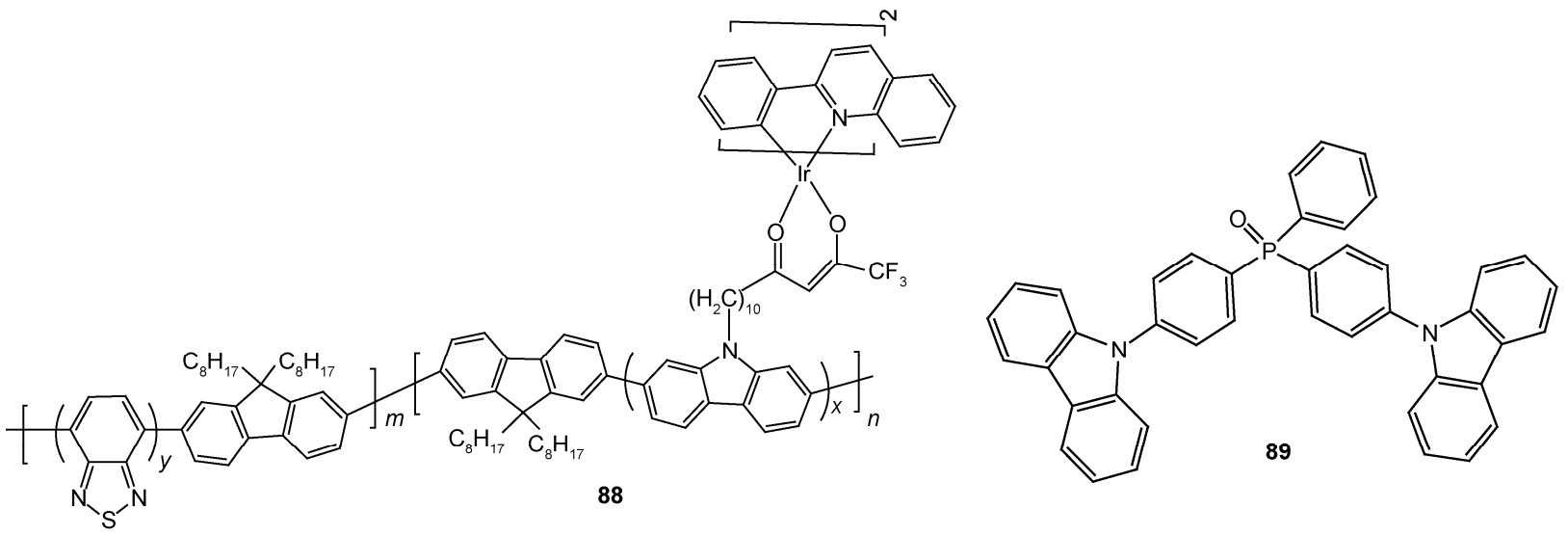

Figure 36

Figure 37 
white-light region and for different applied voltages [86].

In the design of highly efficient phosphorescent devices, the selection of the proper material for each layer is of great importance, because it fundamentally affects the efficiency of energy transfer from the host to the dopant. The use of bipolar materials as hosts for organic electrophosphorescence devices can improve carrier mobilities and balance electron and hole fluxes in the emissive layer. However, a drawback is that the introduction of an electron-donating and -withdrawing moiety to the host molecule leads to an intramolecular charge transfer that reduces the energy gap of the molecule. Cheng et al. synthesized the $p$ - $n$ diblock host material 90 containing a phosphine oxide and two CBZ groups (Figure 37), and this material exhibits a high triplet energy gap of $3.01 \mathrm{eV}$. The material can be used as a universal host for blue, green, and red phosphorescent devices, all giving extremely high efficiencies with turn-on voltages below $3 \mathrm{~V}$ [87].

\section{Concluding remarks}

In the investigation of functional organic EL materials, the relationship between the organic molecular structures and optoelectronic functions should first be established [88,89]. Of the three basic red, green and blue colors, the efficiency, color purity, and lifetime of the first two have almost met the commercialization requirements for full color displays [90,91], whereas the blue light still lags far behind. This difficulty constitutes a bottle-neck that strongly challenges academic and industrial researchers [92,93]. Therefore, it is very important to summarize some rules through a great deal of investigations to further guide the synthesis of new blue light-emitting materials with improved optoelectronic characteristics. During the last two decades, organic electroactive materials have been tested as the active material in a vast array of optoelectronic devices because these materials promise low-cost, flexible, and easily manufactured systems [94-96]. The $p$ - $n$ diblock molecular design concept developed by Huang et al. has produced several series of conjugated polymers and oligomers, whose HOMO and LUMO energy levels, emissive wavelength, and other optoelectronic properties can be tuned in a wide range, which exhibit improved PL and EL properties. However, in addition to stringent prerequisites in terms of optical and CT properties, $\pi$-conjugated molecules designed as active materials for electronic and photonic devices must combine processability and high environmental and photochemical stability, which is an area $p$ - $n$ diblock molecules are still weak [97-99]. Once the above problems are completely resolved, all solid-state OLED devices may fulfill their promise of great utility for people everywhere in the near future.

This work was supported by the National Basic Research Program of China (2009CB930601), the Key Project of the Ministry of Education
(104246), the National Natural Science Foundation of China (20774043, 60706017 and 20574012), the Creative Research Group of Jiangsu College Council (TJ207035 and TJ209035), and the Natural Science Foundation of Jiangsu College Council (10KJB150012).

1 Grimsdale A C, Chan K L, Holmes A B, et al. Synthesis of light-emitting conjugated polymers for applications in electroluminescent devices. Chem Rev, 2009, 109: 897

2 Jiang H J, Wan J H, Huang W. Progress in long wavelength emission in fluorene-based eltroluminescent blue materials. Sci China Ser B, 2008, 51: 497

3 Scherf U, List E J W. Semiconducting polyfluorenes-towards reliable structure-property relationships. Adv Mater, 2002, 14: 477

4 Akcelrud L. Electroluminescent polymers. Prog Polym Sci, 2003, 28: 875

5 Ullrich M, Peter B. The electroluminescence of organic materials. J Mater Chem, 2000, 10: 1471

6 Miyaura N, Suzuki A. Palladium-catalyzed cross-coupling reactions of organoboron compounds. Chem Rev, 1995, 95: 2457

7 Shu C F, Dodda R, Jen A K Y, et al. Highly efficient blue-light-emitting diodes from polyfluorene containing bipolar pendant groups. Macromolecules, 2003, 36: 6698

8 Michinobu T, Okoshi K, Shigehara K, et al. Band-gap tuning of carbazole-containing donor-acceptor type conjugated polymers by acceptor moieties and $\pi$-spacer groups. Polymer, 2008, 49: 192

9 Yu W L, Meng H, Huang W, et al. Tuning redox behavior and emissive wavelength of conjugated polymers by $p-n$ diblock structures. J Am Chem Soc, 1998, 120: 11808

10 Huang W, Yu W L, Meng H, et al. New series of blue-light-emitting polymers constituted of 3-alkylthiophenes and 1,4-di(1,3,4-oxadiazolyl)phenylene. Chem Mater, 1998, 10: 3340

11 Yu W L, Meng H, Huang W, et al. Synthesis of 1,4-bis(1,3,4-oxadiazol-2-yl)-2,5-dialkoxybenzene-oligothiophene copolymers with different emissive colors: Synthetically tuning the photoluminescence of conjugated polymers. Chem Commun, 1998, 18: 1957

12 Yu W L, Meng H, Huang W, et al. Synthesis and characterization of a new $p$ - $n$ diblock light-emitting copolymer. Macromolecules, 1998, 31: 4838

13 Huang W, Meng H, Yu W L, et al. A new blue light-emitting polymer containing substituted thiophene and an arylene-1,3,4-oxadiazole moiety. Adv Mater, 1998, 10: 593

14 Leung M K, Yang C C, Chiu C F, et al. The unusual electrochemical and photophysical behavior of 2, 2'-bis(1,3,4-oxadiazol-2- yl)biphenyls, effective electron transport hosts for phosphorescent organic light emitting diodes. Org Lett, 2007, 9: 235

15 Grazuleviciusa J V, Strohrieglb P, Pielichowski K, et al. Carbazole-containing polymers: Synthesis, properties and applications. Prog Polym Sci, 2003, 28: 1297

16 Knölker H J, Reddy K R. Isolation and synthesis of biologically active carbazole alkaloids. Chem Rev, 2002, 102: 4303

17 Morin J F, Leclerc M, Siove A, et al. Polycarbazoles: 25 years of progress. Macromol Rapid Commun, 2005, 26: 761

18 Meng H, Chen Z K, Huang W. Spectroscopic and electrochemical study of a novel blue electroluminescent $p-n$ diblock conjugated copolymer. J Phys Chem B, 1999, 103: 6429

19 Meng H, Chen Z K, Huang W, et al. Synthesis and characterization of a novel blue electroluminescent polymer constituted of alternating carbazole and aromatic oxadiazole units. Phys Chem Chem Phys, 1999, 1: 3123

20 Huang W, Chen Z K, Lai Y H, et al. A novel series of $p$ - $n$ diblock light-emitting copolymers based on oligothiophenes and 1,4-bis(oxadiazolyl)-2,5-dialkyloxybenzene. Macromolecules, 1999, 32: 118

21 Kang B S, Jun Y S, Shin S C, et al. Wavelength tuning of light-emitting polyarenes via an m-phenylene interrupting block: $\pi-\pi^{*}$ band-gap adjustment of thiophene-based conjugated polymers. Chem Commun, 1996, 10: 1167.

22 Meng H, Huang W. Novel photoluminescent polymers containing 
oligothiophene and m-phenylene-1,3,4-oxadiazole moieties: Synthesis and spectroscopic and electrochemical studies. J Org Chem, 2000, 65: 3894

23 Wang L H, Chen Z K, Huang W. A novel conjugated polymer containing alternating $p$ - and $n$-type moieties with balanced properties of conducting holes and electrons. Macromol Rapid Commun, 2000, 11: 897

24 Xiao Y, Yu W L, Huang W. A novel series of copolymers containing 2,5-dicyano-1,4-phenylene-vinylene-synthetic tuning of the HOMO and LUMO energy levels of conjugated polymers. Chem Eur J, 2000, 6: 1318

25 Chen $\mathrm{Z}$ K, Meng $\mathrm{H}$, Huang W, et al. Photoluminescent poly( $p$ phenylenevinylene)s with an aromatic oxadiazole moiety as the side chain: Synthesis, electrochemistry, and spectroscopy study. Macromolecules, 1999, 32: 4351

26 Meng H, Yu W L, Huang W. Facile synthetic route to a novel electroluminescent polymer-poly(p-phenylenevinylene) containing a fully conjugated aromatic oxadiazole side chain. Macromolecules, 1999, 32: 8841

27 Chen Z K, Huang W, Lee S T, et al. A family of electroluminescent silyl-substituted poly(p-phenylenevinylene)s: Synthesis, characterization, and structure-property relationships. Macromolecules, 2000, 33: 9015

28 Chen Z K, Wang L H, Huang W, et al. Intense green light from a silyl-substituted poly(p-phenylenevinylene)-based light-emitting diode with air-stable cathode. Phys Chem Chem Phys, 1999, 1: 3789

29 Wang L H, Kang E T, Huang W. Synthesis, spectroscopy, and electrochemical properties of a novel $p$ - $n$ diblock poly(p-phenylenevinylene)-related copolymer containing bipyridine. Polymer, 2001, 42: 3949

30 Liu S Y, Chen Z K, Huang W, et al. Novel blue photoluminescent copolymers containing bipyridine and organosilicon. Synth Met, 2000, 114: 101

31 Klärner G, Scott J C, Miller R D, et al. Colorfast blue-light-emitting random copolymers derived from Di-n-hexylfluorene and anthracene. Adv Mater, 1998, 10: 993

32 List E J W, Guentner R, Scherf U, et al. The effect of keto defect sites on the emission properties of Polyfluorene-type materials. Adv Mater, 2002, 14(5): 374

33 Romaner L, Pogantsch A, List E J W, et al. The origin of green emission in Polyfluorene-based conjugated Polymers: On-chain defect fluorescence. Adv Funct Mater, 2003, 13: 597

34 Chen R F, Zhu R, Huang W, et al. A general strategy for the facile synthesis of 2,7-dibromo-9-heterofluorenes. Org Lett, 2006, 8: 203

35 Chen R F, Zhu R, Huang W, et al. Synthesis, structure, and optoelectronic properties of phosphafluorene copolymers. Org Lett, 2008, 10: 2913

36 Yu W L, Pei J, Huang W. Spiro-functionalized polyfluorene derivatives as blue light-emitting materials. Adv Mater, 2000, 12: 828

37 Zhu R, Wen G A, Huang W. Di-channel polyfluorene containing spiro-bridged oxadiazole branches. Macromol Rapid Commun, 2005, 26: 1729

38 Xin Y, Wen G A, Huang W. Hyperbranched oxadiazole-containing polyfluorenes: Toward stable blue light PLEDs. Macromolecules, 2005, 38: 6755

39 Tang D F, Wen G A, Huang W, et al. Photocrosslinkable hyperbranched polyfluorenes containing oxadiazole: Synthesis, photophysics and electroluminescence. Polym Int, 2008, 57: 1235

40 Ma Z, Lu S, Wang P, Huang W, et al. Syntheses, characterization, and energy transfer properties of benzothiadiazole-based hyperbranched polyfluorenes. Polymer, 2006, 47: 7382

41 Wen G A, Peng B, Huang W, et al. Hyperbranched triazinecontaining polyfluorenes: Efficient blue emitters for polymer lightemitting diodes (PLEDs). Polymer, 2007, 48: 1824

42 Jiang H J, Gao Z Q, Huang W, et al. Novel photoluminescent polymers containing fluorene and 2,4,6-triphenyl pyridine moieties: Effects of noncoplanar molecular architecture on the electro-optical properties of parent matrix. Polymer, 2008, 49: 4369

43 Deng L, Furuta P T, Fréchet J M J, et al. Living radical polymeriza- tion of bipolar transport materials for highly efficient light emitting diodes. Chem Mater, 2006, 18: 386

44 Moad G, Rizzardo E, Thang S H. Radical addition-fragmentation chemistry in polymer synthesis. Polymer, 2008, 49: 1079

45 Zhao P, Ling Q D, Huang W, et al. Reversible addition-fragmentation chain transfer polymerization of methacrylates containing hole- or electron-transporting groups. J Polym Sci Part A: Polym Chem, 2007, 45: 242

46 Liu C B, Zhao P, Huang W. New oxadiazole derivatives as promising electron transport materials: Synthesis and characterization of thermal, optical and electrochemical properties. Cent Eur J Chem, 2007, 5: 303

47 Ling Q D, Kang E T, Huang W, et al. Synthesis and nearly monochromatic photoluminescence properties of conjugated copolymers containing fluorene and rare earth complexes. Macromolecules, 2003, 36: 6995

48 Liu S J, Huang C H, Huang W. $\pi$-Conjugated chelating polymers with charged iridium complexes in the backbones: Synthesis, characterization, energy transfer, and electrochemical properties. Chem Eur J, 2006, 12: 4351

49 Liu S J, Wang L H, Huang W, et al. $\pi$-Conjugated chelating polymers with a charged iridium complex in the backbones: Toward saturated-red phosphorescent polymer light-emitting diodes. J Phys Chem C, 2007, 111: 1166

50 Liu S J, Zhao Q, Huang W, et al. Polyfluorenes with on-chain metal centers. Adv Polym Sci, 2008, 212: 125

51 Deng Y, Wang L H, Huang W, et al. Synthesis and characterization of red phosphorescent-conjugated polymers containing charged iridium complexes and carbazole unit. Synth Met, 2007, 157: 813

52 Kulkarni A P, Kong X X, Jen S A. High-performance organic light-emitting diodes based on intramolecular charge-transfer emission from donor-acceptor molecules: Significance of electron- donor strength and molecular geometry. Adv Funct Mater, 2006, 16: 1057

53 Wan J H, Feng J C, Huang W, et al. New $p$-n diblock and triblock oligomers: Effective tuning of HOMO/LUMO energy levels. Tetrahedron Lett, 2006, 47: 2829

54 Zhao P, Ma D G, Huang W, et al. Highly efficient red electroluminescence induced by efficient electron injection and exciton confinement. Synth Met, 2006, 156: 763

55 Roncali J, Leriche P, Cravino A. From one- to three-dimensional organic semiconductors: In search of the organic silicon? Adv Mater, 2007, 19: 2045

56 Wang H Y, Feng J C, Huang W, et al. Cruciform $p$ - $n$ diblock conjugated oligomers for electroluminescent applications. New J Chem, 2006, 30: 667

57 Wang H Y, Wan J H, Huang W. Synthesis and characterization of cross-shaped $p-n$ diblock oligomers. J Polym Sci Part A: Polym Chem, 2007, 45: 1066

58 Jiang H J, Wei W, Huang W, et al. Two novel oligomers based on fluorene and pyridine: Correlation between the structures and optoelectronic properties. J Polym Sci Part A: Polym Chem, 2008, 46: 1548

59 Kido J, Okamoto Y. Organo lanthanide metal complexes for electroluminescent materials. Chem Rev, 2002, 102: 2357

$60 \mathrm{Xu} \mathrm{H}$, Wang L, Huang W, et al. Application of chelate phosphine oxide ligand in Eu-III complex with mezzo triplet energy level, highly efficient photoluminescent, and electroluminescent performances. J Phys Chem B, 2006, 110: 3023

$61 \mathrm{Xu} \mathrm{H}$, Yin K, Huang W. Highly improved electroluminescence from a series of novel Eu-III complexes with functional single-coordinate phosphine oxide ligands: Tuning the intramolecular energy transfer, morphology, and carrier injection ability of the complexes. Chem Eur J, 2007, 13: 10281

$62 \mathrm{Xu} \mathrm{H}$, Yin K, Huang W. Comparison of the electrochemical and luminescence properties of two carbazole-based phosphine oxide Eu-III complexes: Effect of different bipolar ligand structures. Chem Phys Chem, 2008, 9: 1752

63 Pan J F, Chen Z K, Huang W. Protonation of bipyridines and their vinylene-phenylene-vinylene derivatives: Theoretical analysis of the 
positive charge effects. J Phys Chem A, 2001, 105: 8775

64 Zeng G, Chua S J, Huang W. Influence of donor and acceptor substituents on the electronic characteristics of poly (fluorenephenylene). Thin Solid Films, 2002, 417: 194

65 Pan J F, Chua S J, Huang W. Tuning redox behavior and emissive wavelength of conjugated polymers by $p-n$ diblock structures - a theoretical investigation. Synth Met, 2000, 110: 85

66 Chen R F, Pan J F, Huang W. Theoretical investigation of the tunable behavior of $p$ - $n$ copolymers based on oligothiophenes and 14-bis(oxadiazolyl)-benzene. J Phys Chem B, 2006, 110: 23750

67 Chen R F, Zheng C, Huang W, et al. Structural, electronic, and optical properties of 9-heterofluorenes: A quantum chemical study. J Comput Chem, 2007, 28: 2091

68 Shan G C, Fan Q L, Huang W, et al. Theoretical study on energy levels and photophysical properties of $p$ - $n$ block oligomers. J Optoelectronics Adv Mater, 2007, 9: 1373

69 Chen R F, Fan Q L, Huang W, et al. Germafluorene conjugated copolymer-synthesis and applications in blue-light-emitting diodes and host materials. Sci China Ser B, 2009, 52: 212

70 Strehmel B, Sarker A M, Neckers D C. Effect of aromatic ring substitution on the optical properties, emission dynamics, and solid-state behavior of fluorinated oligophenylenevinylenes. J Am Chem Soc, 1999, 121: 1226

71 Facchetti A, Yoon M H, Marks T J. Building blocks for n-type organic electronics: Regiochemically modulated inversion of majority carrier sign in perfluoroarene-modified polythiophene semiconductors. Angew Chem Int Ed, 2003, 42: 3900

72 Huang T H, Lin J T. Tunable dipolar acenaphthopyrazine derivatives containing diphenylamine. Chem Mater, 2004, 16: 5387

73 Chen S Y, Xu X J, Liu Y Q. Synthesis and characterization of n-type materials for non-doped organic red-light-emitting diodes. Adv Funct Mater, 2005, 15: 1541

74 Liou G S, Hsiao S H, Yen $\mathrm{H} \mathrm{J}$, et al. A new class of high $\mathrm{T}_{\mathrm{g}}$ and organosoluble aromatic poly(amine-1,3,4-oxadiazole)s containing donor and acceptor moieties for blue-light-emitting materials. Macromolecules, 2006, 39: 6036

75 Ichikawa M, Kawaguchi T, Taniguchi T K Y, et al. Bipyridyl oxadiazoles as efficient and durable electron-transporting and hole-blocking molecular materials. J Mater Chem, 2006, 16: 221

76 Xu X J, Chen S Y, Liu Y Q. High-efficiency blue light-emitting diodes based on a polyphenylphenyl compound with strong electron-accepting groups. Adv Mater, 2007, 19: 1281

77 Qian G, Zhong Z, Ma D G, et al. Simple and efficient near-infrared organic chromophores for light-emitting diodes with single electroluminescent emission above $1000 \mathrm{~nm}$. Adv Mater, 2009, 21:111

78 Natera J, Otero L, Sereno L. A novel electrochromic polymer synthesized through electropolymerization of a new donor-acceptor bipolar system. Macromolecules, 2007, 40: 4456

79 Zabel P, Dittrich T, Otero L, et al. Engineering of gold surface work function by electrodeposition of spirobifluorene donor-acceptor bipolar systems. Org Electron, 2009, 10: 1307

80 Tonzola C, Alam M M, Jen S A. A new synthetic route to soluble polyquinolines with tunable photophysical, redox, and electrolumi- nescent properties. Macromolecules, 2005, 38: 9539

81 Zhu Y, Champion R D, Jen S A. Conjugated donor-acceptor copolymer semiconductors with large intramolecular charge transfer: Synthesis, optical properties, electrochemistry, and field effect carrier mobility of thienopyrazine-based copolymers. Macromolecules, 2006, 39: 8712

82 Kido J, Okamoto Y. Organo lanthanide metal complexes for electroluminescent materials. Chem Rev, 2002, 102: 2357

83 Karabunarliev S, Bittner E R. Electroluminescence yield in donor-acceptor copolymers and diblock polymers: A comparative theoretical study. J Phys Chem B, 2004, 108: 10219

84 Chen X W, Liao J L, Chen S A. High-efficiency red-light emission from polyfluorenes grafted with cyclometalated iridium complexes and charge transport moiety. J Am Chem Soc, 2003, 125: 636

85 Kim S H, Seo J W, Park S Y, et al. White luminescence from polymer thin films containing excited-state intramolecular proton-transfer dyes. Adv Mater, 2005, 17: 2077

86 Jiang J X, Xu Y H, Cao Y. High-efficiency white-light-emitting devices from a single polymer by mixing singlet and triplet emission. Adv Mater, 2006, 18: 1769

87 Chou H H, Cheng C H. A highly efficient universal bipolar host for blue, green, and red phosphorescent OLEDs. Adv Mater, 2010, 22: 2468

88 Bernius M T, Inbasekaran M, Wu W S, et al. Progress with lightemitting polymers. Adv Mater, 2000, 12: 1737

89 Tao F, Bernasek S L, Xu G Q. Electronic and structural factors in modification and functionalization of clean and passivated semiconductor surfaces with aromatic systems. Chem Rev, 2009, 109: 3991

90 Kabra D, Narayan K S. Direct estimate of transport length scales in semiconducting polymers. Adv Mater, 2007, 19: 1465

91 Wen W, Yu J S, Hu Y, et al. Red organic light emitting device with improved performance using a novel fluorescent dye codoped with phosphorsensitizer. Chinese Sci Bull, 2010, 55: 2738

92 Jiang H J, Feng J C, Huang W, et al. Progress in fluorene-based electrolumineseent materials. Prog Chem, 2005, 17: 818

93 Meier H. Conjugated oligomers with terminal donor-acceptor substitution. Angew Chem Int Ed, 2005, 44: 2482

94 Kelley T W, Baude P F, Theiss S D, et al. Recent progress in organic electronics: Materials, devices, and processes. Chem Mater, 2004, 16: 4413

95 Li R J, Hu W P, Zhu D B, et al. Micro- and Nanocrystals of organic semiconductors. Acc Chem Res, 2010, 43: 529

96 Liu J Q, Chen S F, Huang W, et al. Organic/polymeric memory and their switching mechanisms (in Chinese). Chinese Sci Bull (Chinese Ver), 2009, 54: 3420

97 Roncali J. Molecular engineering of the band gap of $\pi$-conjugated systems: Facing technological applications. Macromol Rapid Commun, 2007, 28:1761

98 Hudson Z M, Wang S. Impact of donor-acceptor geometry and metal chelation on photophysical properties and applications of triarylboranes. Acc Chem Res, 2009, 42:1584

99 Jiang H J. Organic ambipolar conjugated molecules for electronics: synthesis and structure-property relationships. Macromol Rapid Commun, 2010, 31: 2007

Open Access This article is distributed under the terms of the Creative Commons Attribution License which permits any use, distribution, and reproduction in any medium, provided the original author(s) and source are credited. 